\title{
Gespräch 17. - 19. Oktober 1980
}

SR: Es geht für mich zunächst prinzipiell um zwei Fragen die Frage nach der Rolle der Sprache und die Frage nach der Rolle des Schriftstellers in seinem Werk.

GR: Ich kann kurz zwei Sachen sagen, bevor wir ansetzen. Die Rolle der Sprache hat sich bei mir stark verändert. Am Anfang war die Sprache für mich ein sehr subjektiv betrachtetes Instrument und ich habe sie angewandt, um meine inneren Schwierigkeiten darzustellen; das war im einstein, das war im Ausbruch des Ersten Weltkriegs, das war im Wille zur Krankheit so, obwohl ich, z. B. im Ausbruch des Ersten Weltkriegs bei zwei Geschichten, nämlich der Titelgeschichte und "How to be a Detective" eigentlich artifiziell vorgegangen bin, so waren das doch mehr oder weniger Erprobungen meiner Verrücktheit, so ungefähr. ${ }^{1}$ Unter ,Verrücktheit' verstehe ich keinen psychologischen Zustand für mich sondern, dass ich eben, ver-rückt' bin innerhalb der Gesellschaft nicht an einem Platz, wo jemand hingehört. Und dieses Gefühl, das habe ich eigentlich schon immer gehabt, ein Gefühl der Fremdheit, nur weil ich lange Zeit eher Schuldgefühle gehabt habe, dass das so ist. Und aus diesem Schuldgefühl heraus habe ich viele Handlungen gesetzt und gemacht, die ich sonst nie getan hätte und das Schreiben war für mich zunächst eine Verschleierung meiner Person, so würde ich das sagen. Im einstein habe ich versucht mit Hilfe

\footnotetext{
${ }^{1}$ die autobiographie des albert einstein, Frankfurt /M.: Suhrkamp, 1972; Der Ausbruch des Ersten Weltkriegs und andere Romane, Frankfurt a. M.: Suhrkamp, 1972; Der Wille zur Krankheit, Frankfurt/M.: Suhrkamp, 1973.
} 
der Sprache diesen Zustand der Fremdheit ästhetisch darzustellen ohne auf soziale Bezüge einzugehen. Es ist einfach so - ein Versteckprozess vor mir selbst.

Ich habe dann in den anderen Geschichten einfach sprachliche Erfahrungen, die ich in diesem Prozess gewonnen habe, mit diesem Schreiben gewonnen habe, angewendet. Z. B. im Ausbruch des Ersten Weltkriegs und dann im Willen zur Krankheit habe ich diese Sprachsituation für mich artifiziell nutzbar gemacht, insofern als ich sie wie eine mathematische Gleichung anwenden konnte. Mit diesem Buch war ich mit meiner Entwicklung nicht zufrieden, weil es mir eine Einbahnstraße zu sein schien und dass ich auf Grund dieser sprachlichen Beschränkung - weil jede Form eine sprachliche Beschränkung ist - auf Grund dieses artifiziellen Stils vieles, was ich schreiben wollte, nicht mehr schreiben konnte. Wenn man das Thema sozusagen immer der Ausdrucksmöglichkeit anpassen muss, dann muss man irgendwo einen Ausweg suchen und ich habe ganz von vorne, sozusagen, wieder angefangen und in meinem Amerikabuch versucht, mit großer Einfachheit und großer Genauigkeit ein Spektrum zu schaffen, das mir ermöglicht, Dinge und Vorgänge zu beschreiben, die für mich wichtig sind und die ich darstellen möchte.

SR: Du hast in einem Interview gesagt, du hättest damals das Gefühl, dass du Bücher einfach fabrizieren könntest und das war für dich eine Art schriftstellerischer Unzufriedenheit. $^{2}$

2 ,Ich habe gemerkt, dass ich Bücher fabrizieren konnte. ... Ich wollte nicht länger reagieren wie ein Fotoapparat, der bloß registriert und abbildet.' Ulrich Greiner, ,Ich will ein Erzähler sein: Porträt des Schrift- 
GR: Ja, das Gefühl, dass ich diesen Stil, der aus einer Notwendigkeit entstanden ist, faktisch, wie ich gesagt habe, mir schon benutzbar gemacht habe. Ich hätte in dieser Form weiter schreiben können, jeden Tag meine paar Zeilen, aber im Prinzip ist es mir in diesem Stil immer schwerer geworden, weil sich ein gewisser Wortrepetismus in mir gebildet hat: auf bestimmte Wortkonstellationen war ich schon fähig zu reagieren. Das war schon wie ein chemischer Prozess: die Worte haben schon wie in einem chemischen Prozess reagiert, haben bestimmte andere Worte angezogen und bestimmte andere Gedanken gebracht. Es wird vielleicht von Außenstehenden, von bestimmten Kritikern diese erste Phase von mir eher als experimentell betrachtet und die zweite Phase als konservativ, um irgend mögliche Ausdrücke zu gebrauchen, und doch ist für mich jedes Buch experimentell. Ich kann keine andere Einstellung zur Arbeit haben als eine experimentelle Einstellung. Für mich ist überhaupt nur die Überprüfung meiner Erfahrungen in der sprachlichen Form, die ich mir für meine Arbeit ausdenke, möglich. Ich könnte niemals sagen, so jetzt habe ich meinen Stil gefunden und jetzt mache ich zwanzig Bücher damit, sondern ich versuche immer neue Methoden anzuwenden, wenn sie vielleicht auch im ersten Augenblick nicht einsichtig sind. Z. B. das letzte Buch, Der stille Ozean, ${ }^{3}$ ist erstens thematisch eine relativ neue Sache für die Gegenwart, weil diese Verwechslung mit dem Heimatroman im deutschen Sprachraum sofort

stellers Gerhard Roth,' Frankfurter Allgemeine Zeitung, 29. Dezember 1976. Nachgedruckt in U. G. Der Tod des Nachsommers: Aufsätze, Porträts, Kritiken zur österreichischen Gegenwartsliteratur, München: Hanser, 1979: S. 158-163.

${ }^{3}$ Der stille Ozean, Frankfurt/M.: S. Fischer, 1980. 
auf der Hand liegt. Im deutschen Sprachraum ist unter dem Nationalsozialismus, aber auch in der Wilhelminischen Zeit, ein Genre entstanden, der Heimatroman, der hat jahrzehntelang die Auseinandersetzung von Künstlern mit dem Thema versperrt. Und es sind die Begriffe belastet, die man zum Schreiben so eines Romans braucht. Ich habe versucht, mich auf diese Begriffe nicht einzulassen, sondern mich eher im Stil der Russen dem zu nähern oder im Stil eines William Faulkners und der Schriftsteller der Südstaaten, die plötzlich ihre Umwelt entdeckt haben ... Carson McCullers oder Sherwood Anderson in Weinsberg Ohio ...

SR: Solche Schriftsteller, die Russen, die Amerikaner sind sozusagen deine literarischen Ahnen.

GR: Ja! In der deutschsprachigen Literatur lese ich wirklich nur Stifter intensiv und immer wieder ... ${ }^{4}$

\section{SR: Der Stifter ist wirklich wichtig für Dich?}

GR: Er ist mir wichtig, ja, aber ich habe nicht dieses Interesse, ich habe nicht diese Philosophie, die der Stifter vertritt. Am Stifter bewundere ich einfach die Genauigkeit und

SR: Die Genauigkeit des Schreibens?

\footnotetext{
${ }^{4}$ Siehe G. R. ,Technik des Naturempfindens gegen Kunst der Technik.' Neue Kronen-Zeitung, (Steiermark-Ausgabe) (Graz), 11. Februar 1973. Nachgedruckt in G. R. Menschen, Bilder, Marionetten - Prosa, Kurzromane, Stücke, Frankfurt/M.: S. Fischer, 1979: S. 49-51.
} 


\section{GR: Ja! Ja!}

\section{SR: Und des Beschreibens?}

GR: Ja, des Schreibens und des Beschreibens - beides. Der Stifter bringt aus winzigen Mikrokosmen jedes Mal die Welt hervor: das ist das Erstaunliche an dem, was er macht! Deshalb finde ich ihn faszinierend. Und für das jetzige Buch, für den Landläufigen Tod, habe ich in den letzten zehn Monaten neben Büchern, die sich mit dem Selbstmord beschäftigen, Die Aufzeichnungen eines Jägers von Turgenev und Moby Dick von Melville immer wieder gelesen.

SR: Der stille Ozean fängt mit einem Motto von Melville an, stammt das aus dem Roman?

GR: Aus einem Brief an einen Freund ist das. Dieser Brief ist abgedruckt in der Winkler Ausgabe, im Nachwort. ${ }^{5}$ Moby Dick habe ich in vier oder fünf verschiedenen Ausgaben und in verschiedensten Übersetzungen und in Hamburg habe ich eine andere gehabt. Hier auf dem Land habe ich eine und Aufzeichnungen eines Jägers besitze ich in drei, vier Ausgaben, weil ich, wenn ich arbeite oder nachdenke, dort, wo ich

5 „Jetzt, da alles mit Schnee bedeckt ist, habe ich hier auf dem Lande das Gefühl, als wäre ich auf See. Morgens, wenn ich aufstehe, schaue ich aus meinem Fenster wie aus dem Bullauge eines Schiffes auf dem Atlantik." Herman Melville, ,Brief an Evert A. Duyckinck', 13.12.1850: "I have a sort of sea-feeling here in the country, now that the ground is covered in snow. I look out of my window in the morning when I rise as I would out of a port-hole of a ship in the Atlantic." Herman Melville: Correspondence, Hrsg. Lynn Horth, Evanston and Chicago: Northwestern University Press, 1993: S. 173. 
bin, das Buch immer wieder lese. Das dritte Buch, das ich schon seit Jahren immer lese, ist Kaltbliitig von Truman Capote und dann natülich auch die von Camus, Der Fremde und Die Pest und von Sartre Die Erzählungen. Immer wieder lese ich auch die Erzählungen von Hemingway.

SR: Und der amerikanische „New Journalism“ - Tom Wolfe, Hunter S. Thompson, Capote ...? ${ }^{6}$

GR: Mit dem Tom Wolfe habe ich sprachlich nicht so viel zu tun, obwohl ich ihn sehr gerne lese, Radical Chic, z. B. Er gefällt mir sehr, aber er hat auf mich keinen Einfluss.

SR: Einfluss - das würde ich auch nicht sagen.

GR: Ich kenne das alles, ich lese das alles, Ich kenne von Mailer alles, was es in deutscher Sprache gibt. Ich kenne das von Capote auch, speziell Kaltblütig. Es gibt andere Arbeiten von ihm, die ich nicht so gern mag, aber in Kaltblütig ist es das Thema, das mich so interessiert. Es ist eine Art Journalismus dabei, ohne dass auch nur in einem Moment die Kunst verleugnet wird. Es könnte genauso gut der Funk sein, das ist das Große an dem Buch. Es ist nirgendwo im schlechten Sinne Journalistisches dabei, auch nicht im besten Sinne Journalistisches, sondern es ist immer Kunst, es ist immer Imagination dabei - in allem, in den nuichternsten

${ }^{6}$ „New Journalism”, ein in den frühen siebziger Jahren von dem amerikanischen Journalisten Tom Wolfe eingeführter Begriff für eine stark durch Fiktion geprägte Form der Reportage. Siehe Tom Wolfe, The New Journalism, Hrsg. Tom Wolfe and E. W. Johnson, New York: Harper and Row, 1973. 
Protokollen, die er wiedergibt. Diese Genauigkeit und Einfachheit, das zieht mich an dem Buch so an. Dasselbe ist auch im Fremden von Camus, das bis in die letzte Banalität hinein einfach ist. Dasselbe ist auch beim Turgenev, Aufzeichnungen eines Jägers, ein völlig unspektakuläres Buch, aber wenn man es länger liest und indem man sich damit beschäftigt, merkt man die ungeheure Meisterschaft von Turgenev. Beim Moby Dick schätze ich den Mut von Melville, alle Fehler zu machen, die auch heute noch als Fehler gelten: z. B. Wechsel der Erzählperspektive: der Captain Ahab schildert, was er sich denkt, was er in der Ich-Form gar nicht könnte usw. Er kümmert sich einfach nicht darum.

SR: Melville entwickelte einen „ozeanischen Stil“ - er hatte keine Angst vor dem Stilbruch.

GR: So ist es, ja! Also, da sind Passagen mit naturwissenschaftlichen Abhandlungen usw. drin und es müsste heute vielen Kritikern in Zeitungen, die an allem etwas auszusetzen haben, ein interessanter Raster sein, die eigenen Kriterien zu prüfen. Die müssten ja alle das Buch als stümperhaft empfinden, weil sie mit diesen Kriterien, die sie anwenden, das Buch überhaupt nicht verstehen können. Beim Melville kann man sagen, dass es durchwegs eine biblische Sprache ist. An ihm schätze ich auch den Mut zum Fragmentarischen innerhalb des Buches. Das ist jetzt für mich besonders für das neue Buch wichtig; ich schreibe einmal eine Seite, einmal drei Seiten, einmal sieben Seiten, einmal sechzig Seiten und dann vielleicht eine kurze wissenschaftliche Abhandlung über das Gebiet, wie viel Einwohner es sind usw. 
SR: Wie entwickelst Du dieses Strukturprinzip? Für mich hat so eine fragmentarische Erzählstruktur viel mit den natürlichen Bewegungen der Zeit, den Jahreszeiten usw. zu tun.

GR: Daš, was Du sagst, das hat auch Goethe in seinem späteren Schreiben als Ziel hingestellt, selbst zur Natur zu werden. Das sehe ich, z. B. auch bei Melville, im Moby Dick, dass die Kunst in Natur übergeht, ein Stadium erreicht hat, dass sie gegenüber natuirlichen Formen fast schon eine Identität aufweist.

SR: Ist das auch bei Stifter der Fall?

GR: Bei Stifter ist es nicht so weit gegangen. Der erste Mann, der diesen ersten großen Riesenschritt zusammengebracht hat, war Melville. Die anderen haben darüber nachgedacht und es versucht, aber der, dem es gelungen ist, war für mich als erster Melville. Das ist das Ungeheuerliche an diesem Buch. Aber das kann man eben nicht der Literatur entnehmen. Die Erkenntnisse, die ein anderer erschrieben hat, früher oder später, und dort weiterschreiben, sonst würde die Literatur, wenn das so wäre, nach Joyce ganz anders ausschauen, als sie in Wirklichkeit ausschaut. Wenn man sich z. B. Der Erwählte von Thomas Mann anschaut im Vergleich zu Ulysses von Joyce, sind das ja Wege, die in der Literatur überhaupt nichts miteinander zu tun haben. Ulysses scheint an dem Mann spurlos vorïbergegangen zu sein. Doch sehr wohl ist beim Döblin, besonders im Berlin Alexanderplatz, eine Ähnlichkeit mit Joyce vorhanden. Das heißt, dass ein Autor immer nur wahlverwandt irgendetwas 
annehmen kann, dass er eine Wahlverwandtschaft zu irgendeinem Stil in irgendeinem Buch fühlt und dessen Sicht trägt. Ich bin immer überzeugt, dass viele Bücher sehr stark auf einen wirken, die man selbst noch gar nicht gelesen hat. Seitdem ich Die Dämonen von Dostojevski, zwanzig, dreiBig Seiten gelesen habe, beschäftige ich mich mit diesem Buch und ich wage es nicht mehr fertig zu lesen, weil es vielleicht nicht das erfüllen kann, was ich mir als Vorbild aus diesem Buch nehme. Ich lese oft nur über die Dämonen - Essays oder so was - und weigere mich das im Buch...

SR: Das genügt?

GR: Nein, das genügt einem gar nicht, aber ich habe Angst, sozusagen, zu lesen, weil ich damit ein Fetisch verliere. Die ungelesenen Dämonen sind für mich ein Fetisch. Jahrelang war für mich Moby Dick ein Fetisch und richtig durchgelesen in einem Zug habe ich es erst heuer und im Sommer ein zweites, drittes Mal. Vorher war das wie ein Fetisch für mich. Auch Die Aufzeichnungen eines Jägers habe ich ein Jahre lang mit mir herumgetragen, indem ich nur die erste Geschichte gelesen habe, ungelesen als Fetisch. Vielleicht liegt es daran, dass es so schwer ist, literarische Fetische zu finden. Die Beeinflussung liegt nicht in der Richtung, dass ich jetzt etwas kopieren möchte, sondern ich stelle mir vor, was in dem Buch für mich als ästhetische Kategorien und sprachliche und inhaltliche Kategorien drinnen sein könnten, die eine Ähnlichkeit mit dem haben, woran ich denke, womit ich mich beschäftige. Manchmal kommt es vor, dass ich es lese und damit ist es abgeschlossen, dann lese ich es nie mehr wieder. 
SR: Sind diese Schriftsteller, wie Hans Wollschläger es gesagt hat, auch Zeitgenossen? ${ }^{7}$

GR: Ja, alle! Das ist klar. Jeder, das ist das Schöne. In der Literatur gibt es eigentlich für mich als Leser keine Zeitunterschiede. In dem Moment, wo sie mich ansprechen, sind sie meine Zeitgenossen. Büchner, z. B. würde ich jetzt von der deutschen Literatur als denjenigen bezeichnen, der mich am meisten interessiert.

SR: Ich habe mich in der letzten Zeit ziemlich viel mit Oswald Wieners Verbesserung von Mitteleuropa beschäftigt. Wiener bezieht sich auf Wittgensteins Philosophische Bemerkungen: „Die Annahme, dass eine Realität außerhalb der Sprache existiere, hatte sich als Illusion entlarvt." 8

GR: Ich glaube, das kann man nicht so in den Raum gestellt übernehmen. Das Interessante an dieser Feststellung ist für mich jetzt, wo Du sie aussprichst, dass sie sich anders anhört, als zu dem Zeitpunkt, wo ich sie gelesen habe - im Zusammenhang. Sie wurde dabei gar nicht so aus dem $\mathrm{Zu}-$ sammenhang gerissen, weil die Annahme in sich sehr klar ist. Die Aussage von Oswald Wiener würde in seinem Werk

\footnotetext{
${ }^{7}$ Hans Wollschläger (1935-2007), deutscher Polyhistor, Übersetzer von u. a. Raymond Chandler, Dashiell Hammett, William Faulkner, James Joyce (Ulysses 1975), Edgar Allan Poe, Mark Twain und Oscar Wilde.

${ }^{8}$ Oswald Wiener (geb. 1935) war Mitglied der aus dem Wiener Art-Club hervorgegangenen individualanarchistischen Wiener Gruppe (19541964). Siehe Oswald Wiener, Die Verbesserung von Mitteleuropa, Rowohlt: Hamburg, 1969. Vorabdrucke dieses sprachkritischen ,Romans' erschienen zwischen 1965 und 1969 in manuskripte, der Zeitschrift des Grazer Forums Stadtpark.
} 
früher eventuell Flaubert getroffen haben. Es ist eine Flaubertsche Schriftstelleraussage, würde ich sagen, und keine naturwissenschaftliche, philosophische Aussage.

SR: Ganz im Gegenteil zu Dir, kann man sagen...

GR: Ich habe schon eine Verwandtschaft zu dieser Flaubertschen Beziehung zum Schreiben. Das ist mir nicht fremd, dieses stundelange Sitzen über Sprache, um nachzudenken, welche Formulierung am natürlichsten ist. Nein, ich glaube, wir denken beide etwas anderes bei diesem Satz, daher kommt das Gespräch ja. Ich sehe in diesem Satz einen Schriftstellersatz.

SR: Du hast Die Verbesserung von Mitteleuropa als einen Roman gelesen und nicht als Philosophie oder...

GR: Weder als das eine noch als das andere. Ich habe das als Dokument eines großartig denkenden Kopfes und schreibenden Schriftstellers genommen. Es ist sehr schwer zu sagen; diese ganzen Klassifizierungen stimmen kaum bei Wiener. Und da fällt mir ein, dass ich jetzt vor kurzem ein Buch von Carpentier gelesen habe - literarische Aufsätze in dem er schreibt, dass immer erst dann, wenn die Frage gestellt wird: „Das ist ja kein Roman?“, eine neue Form von Roman überhaupt entstanden ist und das Romanschreiben eigentlich darin besteht, neue Formen zu erfinden. Die Verbesserung von Mitteleuropa ist natürlich ein spektakuläres Beispiel für diese These.

SR: Dies ist ein Flaubertsches Prinzip? 
GR: Ja, und ich muss dazu sagen, um dies jetzt auf meine eigene Arbeit umzumünzen, wo ich dieselben Anforderungen stelle. Da fällt mir eigentlich die Kritik von Günter Blöcker in der F.A.Z. ein, der geschrieben hat: „Was soll das sein Der stille Ozean? Ist das jetzt ein Biologieunterricht oder ist das jetzt ein Heimatroman oder ist das ein Bericht vom Land? ${ }^{\star 9}$ Die Fragen kommen nur deswegen von ihm, weil er nach einem Raster gesucht hast, den er darüber legen wollte, weil er aus dem Begriff „Roman“, aus diesem Umfeld gekommen ist und, weil im Stillen Ozean die formalen Dinge, die versteckt sind im Sprachlichen und im Aufbau, eben nicht spektakulär ersichtlich sind, sondern vorgeben, sie seien nicht hier. Das Buch gibt vor, es gebe nur zufällige Bewegungen, die von mir aufgezeichnet werden, als ob alles beliebig sei. In Wirklichkeit - und das hat eben Blöcker ihm vorgeworfen - durchzieht das ganze Buch ein Geflecht des Todes, der Gewalt, der Tollwut, und wenn man das nicht erkennt oder erkennen will, dann kommt man freilich zu einem Missverständnis. Das war die große Überraschung für mich bei den Kritiken des Buches. Ich habe mich Jahre damit beschäftigt, so lang mich damit auseinandergesetzt, dass dieser Prozess des Feinen, des Genauen für mich eine Selbstverständlichkeit geworden war. Ich habe mich einem ganz bestimmten Bild annähern wollen, einer ganz bestimmten Struktur annähern wollen - sprachlich - und erstaunt hat

${ }^{9}$ Günter Blöcker, ,Ein kundiger Protokollant seelischer Irritationen'. In Frankfurter Allgemeine Zeitung, 22. März 1980: „Immer wieder fragt man sich, was das soll. Sind wir in einem Biologiekurs? Ist dies eine Einführung in die bäuerliche Sozialkunde? Ein Leitfaden für Städter, die sich auf dem Lande einrichten wollen? Oder ein Handbuch der österreichischen Folklore?" 
mich, dass eben einige, eigentlich ïberraschend viele, ihm nicht folgen können und das auch nicht erkannt haben. Das hat der Raddatz wiederum sehr richtig erkannt an dem Buch in der Zeit Kritik. ${ }^{10}$ Da hat der Kritik vielleicht etwas gemangelt, dass er sie als Antwort auf Blöcker hin verfasst hat und dadurch sind gewisse andere Aspekte des Buches nicht so deutlich herausgearbeitet. Es wäre schöner gewesen, wenn Raddatz unabhängig von dem Blöcker einfach seine eigene Kritik über dieses Buch geschrieben hätte, die sich nicht als Antwort auf irgendwas versteht, was schon vorher geschrieben ist. Aber im Wesentlichen hat er den Irrtum gezeigt, der Blöcker unterlaufen war.

Aber wir sind vom Thema abgekommen. Ich wollte damit nur sagen, dass die Erneuerung einer Stilform, z. B. die des Romans, unspektakulär aussehen kann. Um ein großes Beispiel zu nennen, in Auf der Suche nach der verlorenen Zeit von Marcel Proust erhält man eigentlich formal nichts Spektakuläres. Es ist einfach ungeheuer, ein gesamtes sprachliches Kunstwerk, aber das Buch hätte ohne weiteres auch hundert Jahre früher geschrieben sein können, das wäre dann auch ein Markstein in der literarischen Entwicklung gewesen. Das zweite ist Der Mann ohne Eigenschaften von Musil, das formal auch nicht so revolutionierend aussieht, aber in Wirklichkeit ist es das natürlich. Genauso wie in den

${ }^{10}$ Fritz J. Raddatz, ,Zeit der Tollwut'. In Die Zeit, 11. April 1980: „Aber Roth schafft dieses Gefühl von Bedrohung ganz ohne deklamatorische Effekte - so haarfein legt er die Schlinge aus, dass selbst ein so sorgsamer Leser (und Kritiker) wie Günter Blöcker zu einem grotesken Fehlurteil kommt. Er sieht in den minuziös hergestellten Landschaftsbildern ,trostlose Langeweile' und landet in seiner Kritik selber in einem trostlosen Mißverständnis ... ". 
letzten Jahren der Einfluss der Südamerikaner, wie z. B. der Marquez, der einen großen Anstoß in der Literatur dargestellt hat. Vorher waren es die Amerikaner, z. B. Truman Capote. In seiner Art ist sein Kaltblïtig sicher ein wesentliches Buch der Literatur.

SR: Du hast mir vorher gesagt, dass das Fotografieren eine Methode ist, sich bewusst zu werden und bewusst zu bleiben.

GE: Wahrzunehmen.

SR: Wahrzunehmen ... und wie ist es mit der Sprache? Ist die Sprache selbst auch eine Methode sich bewusst zu werden und dann bewusst zu bleiben?

GR: Ja, das ist ganz sicher so! Es gibt ja niemanden, der fortlaufend in sich formuliert. Das würde die menschliche Kraft übersteigen, nicht? Der Zustand des sprachlichen Formulierens, bewussten Formulierens - unterbewusst geht dieser Prozess natiirlich schon vor sich - ist wie auch der bewusste Wahrnehmungsvorgang etwas Ermüdendes, der im Alltag trotz allem eine gewisse Ausnahmeerscheinung ist. Wenn ich vier bis fünf Stunden fotografiere, bin ich sehr müde nachher. Ich kann also gar nicht unendlich lang diesen Konzentrationszustand beibehalten - das verlangt sehr große Aufmerksamkeit. Man muss die Landschaft, die Menschen, die Umwelt immer sehr bewusst in sich aufnehmen, dauernd, und darum gibt's auch den Ermüdungsprozess beim Schreiben, darum kann man nicht dauernd fortschreiben, weil dieses dauernd In-Sprache-Fassen eben etwas sehr phy- 
sisch und geistig Ermüdendes ist. Und für mich hängen die beiden Dinge eng zusammen, das Wahrnehmen und das InSprache-Fassen. Das Wahrnehmen ist schon das erste Ausleseprinzip sozusagen. Da muss schon das erste Wichtige passieren, da muss man schon das Wesentliche erkennen, finden und bei sich notieren oder in einem Notizbuch notieren oder mit einem Fotoapparat aufnehmen. Der zweite Prozess ist dann dieses bewusste Ausleseverfahren sprachlicher Natur, wobei es oft zu Überraschungen kommt am Schreibtisch selber. Dies ist ja nicht der Zustand der Erhabenheit über die Arbeit, dass man sich hinsetzt und nach einem inneren Gesetz uiber die Dinge herrscht, die man beschreibt, sondern das wirkt ja wieder zurück. Erst wenn man so in seiner Arbeit vertieft ist - man könnte es fast einen Trancezustand nennen - zwischendurch, ja, nicht immer, dann ist es eine befriedigende Arbeit, dann ist es wahrscheinlich auch etwas, das gut wird - um sich einem Zustand überlassen, wo man nicht weiß, ob das Eis, auf das man sich begibt, auch tragen wird. Was nun das Fotografieren betrifft, da kann ich mit einem Zitat von Ortega y Gasset dienlich sein und zwar schreibt er über die Jagd und sucht, was nun wohl die Faszination an der Jagd sei. ${ }^{11}$ Er geht alle Punkte durch, die man der Jagd vorwirft oder unterstellt oder die man als Motiv sieht und kommt am Schluss auf den Gedanken, dass es nur einen wirklichen, wahren Grund für die ewige Jagdgeschich-

\footnotetext{
11 Ortega y Gasset (1883-1955), spanischer Philosoph. Die deutsche Übersetzung von Gassets Begleitaufsatz zur Jagdgeschichte von Conde de Yebes, Prólogo a „Veinte años de caza mayor”, die im Jahre 1957 unter dem Titel Meditationen über die Jagd als Band 42 in Rowohlts deutscher Enzyklopädie erschien, erregte im deutschsprachigen Raum einiges Aufsehen.
} 
te der Menschheit gibt und zwar den Zustand der Wachheit, dass der Jäger sich bei der Jagd in dem Zustand der absoluten Wachheit befindet - das Ohr, das Auge, die Sinne sind total geschärft, und daran erkenne ich, wie dumpf man normalerweise dahinlebt. Dieser Zustand der enormen Wachheit ist fast wieder ein tranceähnlicher Zustand. Diese ganz enorme Wachheit bringt wieder so etwas Magisches mit sich wie in einem Traum. Man geht ja sonst halb bewusst oder in Gedanken, mit irgendeiner Absicht oder einem Ziel durch die Natur und nimmt mehr wahr und da wird plötzlich das Tun, das augenblickliche Tun in den Mittelpunkt der gesamten Überlegungen gestellt. Alles, was jetzt passiert rundherum ist ja von Wichtigkeit und wird miteinbezogen. Darum gehen ja auch die meisten Jäger am liebsten allein auf die Jagd, weil das Gesellschaftliche diese Wachheit dann nicht zulässt. Ich wollte mit diesem Zitat nur kurz auf den Zustand der Wachheit beim Fotografieren kommen. Das ist etwas Ähnliches, nicht? Ich habe deshalb auf diesen Zustand der Wachheit auch schon im einstein hingewiesen. Ich möchte eine Droge, die mich ungeheuer nüchtern macht und nicht in den Rauschzustand bringt, sondern ins Gegenteil und damit ist ja gleichzeitig wieder ein Rauschzustand da. Das ganze Einsteinsystem ist aus eigentlichen naturwissenschaftlichen Erkenntnissen eines Wahnsinnigen irgendwo entstanden. Diese Ähnlichkeit zwischen der Geisterwelt der Naturwissenschaften mit den ganzen Atomen und Molekülen und die ganzen naturwissenschaftlichen Erklärungen der Natur und der Umwelt ist ja schon wieder etwas Phantastisches. 
SR: Ist das Fotografieren eine Art Bewusstseinsveränderung? Ist es eine Möglichkeit das eigene Bewusstsein $\mathrm{zu}$ verändern?

GR: Alles worauf Du Dich einlässt, verändert Dich ohnedies, aber ich mache das, damit ich dort, wo ich mich aufhalte und wo ich Gedanken niederzuschreiben habe, um sozusagen in meinem Beruf zu bleiben, und dauernd zu sehen und in der Fortsetzung auch sprachlich zu formulieren. Du hast gesehen, ich mache sehr viele Notizen und dies ist für mich ungeheuer wichtig. Ich verwende beiweitem nicht die Hälfte der Notizen, die ich mache, aber es gibt mir einen Zustand der Zufriedenheit, dass ich überhaupt schreibe. Für mich ist das Schreiben unter anderem eine Form der Selbstberuhigung. Wenn ich mich eine bestimmte Zeit am Tag mit dem Schreiben beschäftigt habe, bin ich selber viel ruhiger, als wenn ich das nicht mache. Es ist kein schlechtes Gewissen oder ...

SR: Angst ...

GR: ... sondern ich fühle mich einfach besänftigt, wenn ich das mache. Das kann eine Stunde sein, das kann eine halbe Stunde sein. Das können auch fünf Stunden sein.

SR: Ich schätze, dieses Gefühl der Beruhigung beim Schreiben hat viel mit dem Zustand der Konzentration selber zu tun.

GR: Ja, und auch mit der Erkenntnis, die man dabei gewinnt, mit der Einsicht, die man gewinnt - wie man weiter 
kommt. Das kann man nicht in Seiten messen. Deshalb habe ich zehn Monate jetzt über das Buch nur nachgedacht. In diesen fast elf Monaten seit November habe ich die $\mathrm{Ge}$ schichte für das Zeitmagazin geschrieben und einen ganz kurzen Artikel über den Kaiser Franz Josef und sonst nichts - das war alles. ${ }^{12}$ Sonst habe ich nur gelesen und über dies nachgedacht. Ich war schon beunruhigt über den Zustand, so lang hat es noch nie gedauert, weil ich den formalen Behälter für die Geschichte nicht gefunden habe. Ich wusste nicht, wo das hineingehört, was ich dann damit mache. Das ist auch Arbeit. Da habe ich mich auch, ohne dass ich jetzt am Schreibtisch geschrieben habe, mit dem Schreiben beschäftigt. Also ich meine das im gesamten Sinn: auch das Fotografieren, das Notieren - alles gehört dazu. Ich glaube, wenn jemand ein wirklicher Künstler ist, dann kann er nichts Unkünstlerisches machen, nicht einmal einen Löffel in die Hand nehmen. Das ist etwas, was komplett in einem drinnen ist.

SR: Kann man das lernen oder ist das ...

GR: Das weiß ich nicht. Das kann ich nicht sagen. Es ist sicher eine - was man sportlich Training nennt - auch eine Übungsform. Es ist die Lust, diese Buntheit und dieses Flackern in sich selber wach zu halten. Das kann man sicher nicht so ab- und anstellen - das ist immer da. Daher stammt auch meine Überlegung, dass die Neigung zum Alkohol bei Schriftstellen relativ groß ist. Es ist immer eine Betäubung.

${ }^{12}$ G. R., ,Land an der Grenze'. Zeitmagazin, 16, 11 . März 1980, S. 1829; ,Kaiserwetter in Wien'. Zeitmagazin, 27, 27. Juni 1980, S. 26-27. 
Der Hemingway, z. B. hat immer Whisky als Belohnung getrunken. So schreibt er. Er ist im Kaffeehaus gesessen und hat sich beim Kellner ein Glas Whisky bestellt. Jetzt hat er noch eine Passage geschrieben und als der Whisky schon am Tisch gestanden ist, hat er die letzten drei, vier Sätze mit einer großen Freude geschrieben, weil der Whisky da gestanden ist. Einerseits ist das ein Gluick, dauernd ein Verwandler zu sein und andererseits die innere Erschöpfung und die Müdigkeit, die damit verbunden ist, von der ich gesprochen habe. Natürlich gibt es Epochen - sozusagen, es kommt einem so lange vor - der Leere, kann man sagen, wo überhaupt nichts in einem ist, gar nichts, wo man ein Tier ist. Aber ich kenne das von Freunden, die man beobachtet, dass man trotzdem Verwandler ist, ohne dass man es halt weiß oder es empfindet oder selbst spürt. Man kann sich innerlich ganz leer vorkommen und trotzdem ist man in seinem Verhalten und in seinem Wesen anders. Das ist kein Kriterium, nicht besser oder schlechter, sondern das ist einfach etwas anderes. Es hat etwas von Verrücktheit, von Fremdheit. Es hat etwas von allgemeinem Weltgefühl. Es hat gleichzeitig etwas von einer großen Nüchternheit. Ich glaube, wenn die Menschen dorthin kommen, dass man es Verwandlung nennen sollte, dass es schon Veranlagung zumindest zum Teil - sein muss, sonst wären alle Selbstmörder geworden, wenn nicht gleichzeitig dieses Schreiben dabei gewesen wäre.

SR: Helmut Eisendle hat viel über den Rausch geschrieben. Der Rauschzustand scheint ihm sehr wichtig zu sein. ${ }^{13}$

13 Helmut Eisendle (1939-2003), österreichischer Psychologe und Schriftsteller. Mitglied der Grazer Autorenversammlung. Siehe insbe- 
GR: Für alle ist er sehr wichtig. Ich kenne kaum einen Autor, der nicht trinkt oder Tabletten nimmt oder sonst irgendwie mit sich selbst experimentiert, um sich zum Schweigen zu bringen zwischendurch. Das wird genauso angewendet, wenn das Schweigen in einem zu groß wird. Das ist wieder die Sehnsucht nach diesem anderen Gefühl, dem schöpferischen Gefühl. So geht dieses Spiel immer hin und her zwischen dem Schöpferischem, dem Schreiben und dem Betäuben und der Leere. Und dort, wo eben der Wechsel gestört ist, kommen die persönlichen Schwierigkeiten für einen Autor.

SR: Das Schreiben, die Sprache ist das denn eine Methode eine andere Ebene des Bewusstseins zu erreichen?

GR: Sicher ist es das auch. Ich meine, es gibt nicht nur ein Motiv für das Schreiben. Es sind viele Motive. Man kann sagen, dass sicherlich ein erotisches Moment eine Rolle spielt beim Schreiben, das einem von ideologischen Kritikern als Selbstbefriedigung hämisch vorgeworfen wird, aber ich glaube, zum Beispiel, dass der Kafka in diesem Sinne ein ziemlich großer Selbstbefriediger war; das sagt für mich nichts aus. Das sind einfach gegenstandslose Vorwürfe. Sicher ist es besser, dass Kafka Selbstbefriediger in der Sprache war, als dass er es nicht getan hätte, nicht!

SR: Was bedeutet das, Erzähler zu werden? Ich beziehe mich hier auf die Schreibkrise, in die Du ungefähr 1972 geraten bist.

sondere seinen Roman Exil oder Der braune Salon - Ein Unterhaltungsroman, Salzburg: Residenz Verlag, 1977. 
GR: Ja, es ist so: prinzipiell Schriftsteller zu sein. Ich glaube, ich war immer einer, ohne es zu wissen, zunächst in der Kindheit. Zum Beispiel habe ich mir nach dem Krieg, 1945 oder so, Bücher gemacht als Kind, selbst gezeichnet. Meine Großmutter hat das zusammengenäht. Ich habe mir eine kleine Bibliothek gemacht, habe Geschichten geschrieben, und es ist eigentlich so fort gegangen bis zu meinem zwölften Lebensjahr. Dann habe ich ein Tagebuch geführt, viele Jahre. Ich habe natülich auch Gedichte gemacht. Damals hatte ich ein ganz dickes Heft davon - Gedichte. Das habe ich verloren und seither habe ich diese Angst vorm Verlieren. Ich nehme es [meine Arbeit] immer mit. Den einstein habe ich auch zwei Jahre lang in einer schwarzen Aktenmappe immer mit mir herumgetragen. Egal, wo auch ich war, nahm ich dieses Buch mit. Und bei dem Ding [Landläufigen Tod] wo ich hingehe habe ich's im Koffer.

SR: Er sieht wie der Koffer eines Arztes aus. Ist das eigentlich so?

GR: Er wurde in einem Müllkübel gefunden, weggeworfen, Sperrmüll. Aber er entspricht meinen Zwecken, genau das Format, die Größe. Ich kann ihn als Handgepäck ins Flugzeug mitnehmen und er passt ganz genau.

Ich habe dieses Gefühl schreiben zu müssen immer gehabt. Ich kann mich überhaupt nicht erinnern an eine Zeit, wo es nicht vorhanden war. Ich würde nicht so einfach sagen, dass es das Anderssein war, das die Ursache war. Ich bin nicht Schriftsteller geworden, wie man Koch oder Fahrradmechaniker wird, sondern ich konnte es gar nicht anders machen so würde ich heute sagen. Ich habe auch gar nicht versucht 
dagegen zu arbeiten, sondern es war für mich immer selbstverständlich.

Ich glaube nicht, dass ich außerhalb der Gesellschaft stehe. Im Gegenteil glaube ich, dass ich sehr intensiv an der Welt teilnehme. Ich habe zehn Jahre im Rechenzentrum gearbeitet: ich war zuerst Operator und habe am Schluss eine Abteilung mit dreißig Leuten gehabt.

SR: Hast Du schon etwas über Deine Erfahrungen beim Rechenzentrum geschrieben?

GR: Indirekt war es der Schluss im Neuen Morgen, wo Dalton sein Leben erzählt. ${ }^{14}$ Wir waren ein Service-Rechenzentrum und sicher möchte ich mich einmal damit beschäftigen, aber ich bin heute noch nicht so weit, dass ich, obwohl es schon Jahre hinter mir liegt, darüber schreiben könnte, so berührt mich das noch immer. Ich bin mit der Erfahrung noch nicht so weit, dass ich sie so verarbeitet habe, dass ich im Augenblick darüber schreiben könnte. Man könnte mir sagen, er hat dort zehn Jahre gearbeitet und er sollte doch über das Rechenzentrum schreiben. Damals war ich so in der Sperre drin, und es hat so einen Einfluss auf mich gehabt, dass ich mich die ganze Zeit widersetzen muss, ja sodass ich heute noch nicht in der Lage bin, das ganz abzusehen, was da wirklich mit mir vorgegangen ist.

SR: Das Schreiben war damals ein richtiges Bedürfnis für Dich.

${ }^{14}$ Ein neuer Morgen, Frankfurt/M.: Suhrkamp, 1976. 
GR: Ja, in der Zeit, da war es ganz stark. Da habe ich einmal ein ganzes Jahr Nachtdienst gehabt und ich habe trotzdem am Tag immer weiter geschrieben. Das kann nicht mein Leben sein, habe ich mir gedacht, das ist nicht, was ich unter meinem Leben verstehe. Ich habe eigentlich nie soziale Ansprüche mit meinem Leben verbunden, nie. Ich habe gedacht, ich muisse ein Auto oder irgendetwas Bestimmtes haben - es macht mir Spaß das eine oder andere - aber ich habe nie einen Besitzdrang in dem Sinne gehabt. Mir geht es um keinen Status oder irgendetwas, sondern mir geht es um die Funktion, um das, was ich brauche für meine Arbeit und dann habe ich es gekauft möglichst schnell. Und am Lande, das Haus, das habe ich immer nur gemietet: es ist nicht mein Eigentum. Es war, wie ich hingezogen bin, ein sehr karges Haus. Es ist ein so genanntes Winzerhaus. ,Winzer' heißen dort die Knechte, woraus hervorgeht, was die für eine Einstellung zum Winzer haben. Ich habe mich heuer im Sommer mit Kindern dort unterhalten. Sie haben mir einen Apfel gegeben und sie haben auf ein Loch gedeutet. Nein, es waren natürlich Kirschen. Da soll ich nicht essen. Da ist ein Winzer drin. Ich habe gefragt, was ist das? Es ist ein Wurm. Und so haben die sie auch leben lassen. Die Zimmer sind von der Größe eines Loches gewesen und haben sechs bis zehn Leute drinnen gehabt. So ein Haus ist es. Das Haus hat ursprünglich zwei Zimmer gehabt, eine Küche und ein Zimmer und da ist auch am Dachboden ein kleines Zimmer gemacht worden. Als ich hingekommen bin, war alles kaputt. Der Verputz ist von der Decke heruntergehängt. Das Wasser war ungenießbar. Der Strom, war abgeschaltet, weil die Rechnungen seit Jahren nicht bezahlt wurden. Ich habe dort wirklich unter harten Umständen mit einem Betteinsatz 
auf dem Küchenboden begonnen zu wohnen und es herrichten zu lassen. Jetzt erst, im vierten Jahr, ist es so weit, dass es wohnlich ist. Es sind zwei Zimmer mit Holz getäfelt. Ich habe mir selber vor zwei Monaten ein Bett gekauft. Ich habe immer auf einem Einsatz auf dem Boden im Schlafsack geschlafen und ansonsten nur das gehabt, was drinnen war in der Wohnung. Ich habe noch einen alten Herd und damit habe ich eingeheizt. Also, das hat mir gepasst, aber ich bin nicht aus ideologischen Gründen oder alternativ auf dem Land. Das war mir zu dumm. Ich bin alternativ auch in der Stadt. Ich bin auch gerne hier in der Wohnung. Ich lebe dort halt mit meiner Literatur zusammen. Das ist es, was mich anzieht. Im Rechenzentrum habe ich die erste Veröffentlichung gehabt; nach dem einstein war nur der Wunsch in mir so schnell wie möglich nicht mehrt dort arbeiten zu müssen. Früher war es wie eine Hoffnung in mir, dass es nicht sein muss, da arbeiten zu müssen. Dann war es eigentlich der Wunsch, dass es jetzt so rasch wie möglich aufhört. Es hat ja dann noch einige Jahre gedauert auf Grund meiner Familiensituation. Es waren noch sechs Jahre, die ich dort war, aber ich habe immer gehofft ...

SR: Hast Du ab und zu das Gefühl gehabt, dass Deine Rolle als Schriftsteller eine Art Schicksal ist, dass es irgendwie unvermeidlich war, Schriftsteller zu werden?

GR: Sicher, aber ich bin nicht in jeder Situation Schriftsteller. Aber es heißt auch nicht, dass ich immer schreibe. Ich könnte mir vorstellen, dass ich fünf Jahre Taxi fahre, aber ich empfinde mich jetzt als Schriftsteller, das heißt... 
SR: Du bleibst trotzdem Schriftsteller.

GR: Ja, oder ich kann auch verschollen sein, oder man kann mir auch die Anerkennung total versagen und ich kann sie mir selber auch versagen. Es bleibt aber immer das Bewusstsein da, entweder als Depression, dass man nicht gut genug ist, oder als etwas, das ich immer aufzeichne, wenn ich etwas bemerke und ich denke mir, das ist jetzt sehr schön, es könnte auch schön zum Schreiben sein, wie etwas sich bewegt hat, oder welche Sätze der gewählt hat ... Das ist, glaube ich, das Wesentliche. Schicksal, würde ich sagen, wäre etwas zu viel, weil der Ausdruck zu kräftig dafür ist. Ich würde eher sagen, dass es eine Eigenschaft ist, so wie es Biologen gibt, die schon als Kinder Schmetterlinge sammeln. Da ist es halt drin. Bei mir war es immer das Lesen und das Schreiben: ich habe gar nicht so gerne gezeichnet wie die anderen Kinder, aber ich habe immer für mich geschrieben. Ich kann mich auch erinnern, ich habe bei allen Zeichnungen Sprechblasen hingeworfen, wenn jemand etwas gesagt hat, ohne dass ich iberhaupt Comicshefte gekannt habe, so einen Einfluss hat es sicher nicht gegeben 45, 46, 47 - 47 war ich fünf Jahre alt und da habe ich das schon gemacht. In der Schule haben wir am 1. November ein Allerheiligenbild gemacht. Da wurde ein Friedhof gemalt, der Grabstein mit einem Schornstein, da wo er sonst geraucht hatte, eine Sprechblase. Ich weiß nicht, warum ich sie darauf gestellt habe, aber ich weiß nur, dass es meinem Lehrer absonderlich vorgekommen ist, dass mit Toten gesprochen wird.

Ich sehe mich nicht anders dadurch, weil ich ein Schriftsteller bin, sondern ich wäre auch als Operator im Rechenzent- 
rum, wenn ich geblieben wäre, eben anders gewesen. Unter dieser Andersartigkeit habe ich immer gelitten. In der Schule bin ich mit vierzehn Jahren oder dreizehn Jahren von der Klasse gequält worden. Es ist über Monate gegangen und seither ist die Schule eben ein großes Drama und ich hasse die Schule. Mir tun die Kinder leid, die in die Schule gehen müssen. Es geht nicht anders, das sehe ich ein, aber ich bedauere sie zutiefst und ich verstehe jedes Kind, das Selbstmord begangen hat in der Schule: mir ist das vollkommen klar. Ich bin mir von der ersten Stunde an in der Schule dumm vorgekommen und ausgeliefert. Alles ist so schnell gegangen in der Schule und den ganzen Gegenständen ... mich hat es gar nicht interessiert.

SR: Dieses Gefühl der Dummheit ist das vielleicht nicht etwas anderes, eine Art Stummheit?

GR: Ja, Dummheit hat aber für mich Geist. Diese soziale Umwelt, die mich umgeben hat, zu der habe ich keine Einstellung gefunden, weil ich mich überhaupt kaum einstellen kann, das ist mein Fehler, meine Schwierigkeit soll man sagen. In gewissen Umweltbereichen fällt das auf. Am Land da gibt es Verrückte und gibt es Säufer und alles: da fällt man nicht auf. Da kann man sich, wenn man nicht zu sehr städtische Verbrechen mit sich bringt, also Haschisch oder so etwas, ziemlich viel erlauben und wenn man den Leuten ein bisschen Menschenliebe entgegenbringt, die ich für sie empfinde. Im Rechenzentrum war es so, dass ich mit den Mitarbeitern, die ich hatte, sehr gern zusammen war, auch privat, wir haben auch unsere Freizeit zusammen verbracht. Es war einfach eine Gruppe, in der ich mich wohl gefühlt 
habe und ich war dort lange Operator, also im Schichtbetrieb für Computerarbeit, das war die geringste Arbeit im Rechenzentrum. Es war nicht, weil ich dort Abteilungsleiter war, sondern einfach, weil ich die Leute gern gehabt habe. Damals wollte ich auch nichts arbeiten, was artverwandt ist, ich wollte nichts arbeiten, was wieder ins Schreiben hineingeht, weil ich gefürchtet habe, dass ich mich dadurch eher ruiniere und dass meine Entwicklung nicht so rein erfolgt, wie sie erfolgt, wenn ich eine andere Arbeit mache. Ich würde heute, wenn ich wieder arbeiten müsste, sicherlich keine Zeitungsarbeit oder so etwas annehmen, sondern höchstens würde ich ein Filmdreher werden, das hat schon fast mehr mit Management zu tun als mit einem selbständigen, freien Schriftstellerdasein.

SR: Wie siehst Du jetzt Deine gesellschaftliche Rolle als Schriftsteller? Was ist Deine Funktion in Bezug auf die Gesellschaft?

GR: Warum nicht zuerst zusammenfassen, wie ich mich im Gesamten sehe. Warum ich Erzähler sein wollte.

Nachdem diese subjektive Phase, von der wir heute schon gesprochen haben, abgeschlossen war beim Schreiben, war das Bedürfnis über Dinge zu schreiben, die mit dem anderen Stil halt nicht möglich waren, nicht so ,sophisticated' sozusagen, nicht nur für literarische Fans zu schreiben, sondern die Frage war, ob es nicht möglich ist, diese Qualität in einer anderen Form ebenso zu verwirklichen: daher dieser Entschluss Erzähler zu werden. Es ist schwer, als Schriftsteller zu leben, gesellschaftlich, trotzdem würde ich nichts anderes machen wollen, aber es gibt kaum einen Beruf, der mit so 
vielen Unterstellungen fertig werden muss, und zwar wehrlos fertig werden muss, wie den des Schriftstellers. Ein Politiker, der sehr hart kritisiert wird, der hat auch seine Zeitungen - damit beschäftigt sich eine ganze Industrie das richtig $\mathrm{zu}$ interpretieren, was aus des Meisters Mund gekommen ist - aber ein Schriftsteller, der hat im Grunde genommen niemanden. Ein Kritiker, der ihm gut gesinnt ist, ist trotzdem aus einer anderen Welt. Es kann daher nur sporadisch eine Form von Verständigung zwischen Schriftsteller und Kritiker geben, weil es eben zwei ganz verschiedene Welten sind, mit ganz verschiednen Ansichten.

SR: Der deutsche Kritiker Ulrich Greiner, der jetzt für Die Zeit uiber Gegenwartsliteratur schreibt, findest Du ihn als Kritiker sympathisch ${ }^{15}$

GR: Ich mag ihn, ja, das sage ich, aber das hängt sicher damit zusammen, dass er positiv über mich geschrieben hat. Ich glaube nicht, dass ich ihn so möchte, wenn er mich verrissen hätte. Ich muss ganz ehrlich sein, es ist auch vollkommen verständlich, wenn ich in der Zeit oder in $\operatorname{der} F$. A. $Z$. eine negative Kritik habe, und wenn jemand eine gute Kritik schreibt und sie stimmt auch noch dazu - also, es kommt das heraus, was man gemeint hat - dann ist es automatisch, dass eine gewisse Sympathie da ist, weil der, der eine Kritik schreibt, die richtig ist, der hat einen ja irgendwo erkannt - man fühlt sich sicher auch anerkannt. Aber es ist nicht die Rolle eines Freundes, das ist nicht möglich. Ich kenne ein oder zwei Kritiker, mit denen ich gut auskomme,

15 Siehe Anmerkung 2. 
aber das ist nur aus großer Entfernung und nur durch sehr sporadisches Sich-Sehen möglich. Für mich ist eine Kritikerfreundschaft so schwer vorstellbar, wie eine Freundschaft für mich in der Schule zwischen mir und dem Lehrer vorstellbar war - so ist das ungefähr. Man freut sich auf die Schule bei diesem Lehrer, weil man einen Einser kriegt, ansonsten möchte man nicht das Leben des Lehrers teilen. Man hat eine andere Vorstellung vom Leben. Ich vergeude auch nicht meine Energien gegenüber Kritikern. Das Beste ist, das, was in den Zeitungen steht, schweigend hinzunehmen. Die Zeit muss arbeiten, man muss das akzeptieren: es hilft gar nichts anderes.

Das zweite Schwere ist die wirtschaftliche Abhängigkeit der Schriftsteller. Die Einnahmen kommen nicht in dem Maße, wie man es sich wünscht. Ich wünsche mir das so, dass ich mich sehr auf die Arbeit konzentrieren kann, die ich mache, dass ich nicht nächtelang wach bleibe aus Sorge um den nächsten Geldbetrag, der kommt. Für mich ist es beim Verlag besser, ich kriege da monatlich Geld. Die Frist steht im Vertrag, aber das sind Vorschüsse, die ich abrechnen muss, und es gibt ja kaum einen arbeitenden Menschen, der, nachdem er z. B. seine Firma gewechselt hat, bei der Firma sein Gehalt zurückzahlen muss, oder einen Teil seines Gehalts: das ist ja unmöglich, das ist wirklich nur bei einem Autor der Fall, oder, z. B. beim Theater, wo man zehn Prozent der Einnahmen bekommt, da verliere ich faktisch von jeder verbilligten Karte noch zehn Prozent. Ich bin der Einzige, der dort wirklich ein Risiko trägt: der Regisseur, die Schauspieler, die Kulissenschieber, die haben alle ihre Gehälter, und wenn das Theater schlecht besucht ist, und ich gar nichts dafür könnte, was ja auch möglich wäre, weil die Auffüh- 
rung z. B. schlecht ist, bin ich der Einzige, der das finanziell zu spüren bekommt. Ein kurzes Beispiel, um das zu erklären. Mein erstes Stuick Lichtenberg ist im Grazer Schauspielhaus hier uraufgeführt worden. ${ }^{16}$ Es haben drei Gäste mitgewirkt in dem Stück. Es haben 80 Personen Platz in der Probebuihne, und die waren ausverkauft - eine Karte hat nur 30 Schilling gekostet - und es konnte nur zehnmal gespielt werden oder achtmal, weil drei Gäste dabei waren, und die sind nach der Verpflichtung wieder gefahren, und dann ist das Stück eingestellt worden. Da habe ich - das kann man sich ausrechnen - zehn Prozent von 30 Schilling sind 3 Schilling, mal 80, sind 240 Schilling am Abend verdient. Das sind $30 \mathrm{DM}$ und zehnmal ist es gespielt worden. Also insgesamt habe ich 300 DM verdient. Davon muss ich ein Drittel an den Verlag abführen. Es bleiben 200 DM übrig und die musste ich noch versteuern. Die 200 DM habe ich aber erst bekommen: ein halbes Jahr oder ein dreiviertel Jahr nachdem das Stück fertig war, ist es im Druck vorgelegen und vom Grazer Schauspielhaus angenommen worden, dann ist ein weiteres halbes Jahr vergangen bis der Aufführungstermin war. Das sind jetzt ein einviertel Jahre. Geschrieben habe ich fast ein Jahr daran - das sind zwei einviertel Jahre, und die Abrechnung vom Theater erfolgte ein halbes Jahr später, nachdem es fertig gespielt war und der Verlag hat nach einem weiteren halben Jahr an mich gezahlt. Die Abrechnung des Verlags an den Autor ist halbjährlich. Damit sind vier Jahre vergangen von dem Zeitpunkt, wo ich das Stuick begonnen habe. Aber inzwischen muss man von etwas leben.

${ }^{16}$ Lichtenberg, Frankfurt/M.: Verlag der Autoren, 1973. 
Natürlich kann man viel verdienen beim Schreiben, aber wenig beim Theater. Der Simmel verdient viel und der Grass. Aber beim Theater z. B., wenn man nur eine Bühne hat, und sei es auch eine große Bühne mit 800 Zuschauern, dass man das Geld hereinkriegt, für ein Jahr Arbeit, ist kaum drinnen, auch wenn das Fernsehen eine Übertragung macht, was ja nicht immer der Fall ist.

SR: Was verdienst Du bei einer Fernsehübertragung?

GR: Hier in Österreich $10.000 \mathrm{DM}$ und ein Drittel geht an den Verlag.

SR: Immer ein Drittel an den Verlag?

GR: Immer ein Drittel. Es bleiben 7.000 DM, und die muss ich versteuern. Also, es bleiben übrig ungefähr 5.000 DM. In Deutschland ist es mehr: das sind 25.000 DM, aber Du musst Dir vorstellen, dass ich mit dem Geld fünf Leute erhalte. Und bei den Erzählungen - ich weiß nicht, ob Dich das interessiert -

SR: Ja, das würde mich bestimmt interessieren.

GR: Für den einstein habe ich 1.000 DM bekommen - eine generelle Abfindung. Für den Künstel 1.000 DM, auch für den Ausbruch des Ersten Weltkriegs. Für Wille zur Krankheit $1.000 \mathrm{DM}$, keinen Groschen mehr! Dann habe ich für den Großen Horizont, nach insgesamt acht oder neun Jahren schriftstellerischer Arbeit, 3.000 DM gekriegt. Ich wollte zu einem anderen Verlag gehen, es wollte mich der Residenz 
Verlag haben für den Großen Horizont und da hat mich Unseld zurïckgehalten und hat mir 4.000 DM für den Großen Horizont gegeben, aber auch das war eine generelle Abfindung - damit war alles erledigt.

Ich habe für das Kinderbuch Herr Mantel und Herr Hernd 1.000 DM gekriegt. ${ }^{17}$ Dann bin ich mit dem Neuen Morgen gekommen und wollte wieder weggehen. Dann habe ich 2.000 DM monatlich von Unseld bekommen, ein Jahr lang, aber nur mit der Auflage, dass dafür die nächsten drei Arbeiten, inklusive dem Neuen Morgen, ihm gehören, aber eben nur für ein Jahr. Nach diesem Jahr müsste ich wieder arbeiten gehen oder so etwas. Ich konnte das nur schaffen, indem ich, was er nicht wusste, das Stück Sehnsucht fast fertig hatte. ${ }^{18}$ Ich war damals noch beim Verlag der Autoren, beim Theaterverlag. Der Unseld, Suhrkamp, wusste nicht, dass ich beim Verlag der Autoren schon ein Stück fertig gehabt habe, und von der Dämmerung ungefähr mehr als die Hälfte fertig gehabt habe. Der Neue Morgen hat als ein Buch gegolten und er hat diesen Vertrag mit mir abgeschlossen, der eigentlich ein selbstmörderischer Vertrag war, weil in einem Jahr drei Arbeiten, das kann man nicht machen, es ist fast unmöglich. Ich hatte aber praktisch schon zwei Sachen, nämlich den Neuen Morgen und Sehnsucht fertig und hatte jetzt ein Jahr Zeit, fast ein Jahr Zeit, um die Dämmerung fertig zu machen und die habe ich relativ schnell fertig gehabt, in ein paar Monaten. Dann bin ich weggegangen von Unseld. Mit der Winterreise bin ich zu Fischer gegangen

${ }^{17}$ Herr Mantel und Herr Hemd, Illustrationen von Ida Szigethy, Frankfurt/M.: Insel, 1974.

${ }^{18}$ Lichtenberg / Sehnsucht / Dämmerung - Stücke, Frankfurt/M.: Fischer Taschenbuch Verlag, 1983 (=Fischer Taschenbuch 7068). 
unter der Bedingung, dass sie wieder alles auflegen. Das Motiv für das Weggehen war jedoch - Unseld wollte mir das Geld weitergeben - weil der Beckermann zurïckgekommen ist. Beckermann war immer mein Lektor beim Suhrkamp bis auf den Neuen Morgen ${ }^{19}$. Er hat mir vom Betreuungmäßigen her am meisten geholfen und er ist vom Suhrkamp Verlag ohne triftigen Grund entlassen worden, damals. Er musste nach Japan - er ist als Professor nach Japan gegangen und ist dann zum Fischer zurückgeholt worden und damit hatte ich wieder das Gefühl, dass ich irgendeinen Arbeitsplatz habe.

Das waren doch immerhin die ersten acht Jahre eines Schriftstellers und es wäre unmöglich gewesen, dass ich davon hätte leben können - so musste ich arbeiten.

SR: Es war wirtschaftlich betrachtet kein Beruf.

GR: Wirtschaftlich ist es aussichtslos gewesen, nicht sehr lukrativ. Und wenn es lukrativ ist, ist die Steuersituation eine Katastrophe. Ich muss in dem Jahr, in dem ich es verdiene, auch versteuern, und wenn ich drei Jahre nichts mache, im vierten Jahr kriege ich es wieder und kann im vierten Jahr alles versteuern. Aber es ist natürlich drinnen, das muss man prinzipiell sagen, siehe Grass oder Böll oder so jemanden, die haben es sicher gar nicht so schlecht - Walser, Frisch. Es ist aber das Schreiben ... Ich würde es nicht verpönen das Geld. Der Heinrich Heine hat sehr viel uiber das Geld nachgedacht, aber mir ist es tatsächlich nicht das We-

${ }^{19}$ Gerhard Roth, Winterreise, Frankfurt/M.: S. Fischer Verlag, 1978. 
sentliche. Ich glaube immer, dass es weitergeht, auch wenn ich nichts verdiene. Zum Beispiel, ich habe keine Pensionsversicherung, weil es einfach nicht mit meiner geistigen Haltung als Schriftsteller vereinbar ist. Und ich möchte auch keine Krankenkasse haben. Die habe ich jetzt nur so lang meine Kinder über mich versichert sind, also bis die studieren. Dann werde ich auch keine Krankenkasse mehr nehmen, weil ich mir im Grunde genommen nicht die Freiheit nehmen möchte, außerhalb der Gesellschaft zu leben, aber mit ihr zu leiden - will sagen - ich möchte, niemandem verpflichtet sein, von niemandem etwas bekommen, was mir vielleicht nicht zusteht. Ich finde die Krankenkasse eine wunderbare Einrichtung, aber für mich ist sie natürlich nicht da. Ich möchte auch nicht in Spitäler usw. Ich finde, was den Künstler betrifft, war die Zeit, z. B. wo der van Gogh gelebt hat und sich erschossen hat, besser, weil er untertauchen hat können in seine Andersartigkeit, weil diese Andersartigkeit schon verbreitet war. Es hat damals ein großes, armes Proletariat gegeben. Das war die Zeit des großen Elends der Landarbeiter, da ist ein Schriftsteller oder Maler oder so etwas nicht weiter aufgefallen ist.

SR: Der van Gogh hat aber auch als Pfarrer gearbeitet.

GR: Ja, aber ich sage nur, er konnte sich verdriicken, sozusagen, und das wird immer schwerer. Es ist alles immer mehr durchdacht und was viel Segen bringt, bringt einem auch gleichzeitig eine noch größere Einengung des Andersartigen. 
SR: Du hast einmal gesagt, schreiben heißt ehrlich sein. Heißt Schriftsteller zu sein ehrlich zu leben?

GR: Ich will sagen, immer den Versuch zu unternehmen, so zu leben. Dass einer ehrlich leben kann und nie lügt, das glaube ich nicht, es ist wohl unmöglich; oder dass einer nicht irgendeine Schwäche hat, oder viele Schwächen hat, die er verbergen will, oder er etwas verleugnet oder ... Natuirlich die eigene Fehlerhaftigkeit ist bei jedem ja auch ein Motor zum Schreiben und ist auch ein Grund, warum er andere fehlerhafte Menschen lieben und verstehen kann. Ich muss nicht meine Fehler, die ich habe, immer öffentlich auch bekennen und alles sozusagen, richtig' (unter Anführungszeichen) zu machen. Alles ,richtig' zu machen ist ja auch wieder eine Form der Unmenschlichkeit. Aber beim Schreibprozess da gibt's nichts als die Wahrheit zu schreiben. Unwahrheit wird ja genug geschrieben. Und es ist immer interessant, das was Egon Erwin Kisch gesagt hat, „Das Faszinierendste ist die Wahrheit" oder so habe ich sinngemäß den Spruch von ihm gehört. Die Wahrheit ist es, die den meisten Ärger hervorruft - und so ist das auch! „Kannst du das auch schreiben?“ aber wenn man sich diese Frage stellt, ist es schon vorbei.

Ich bin ein Angeklagter beim Schreiben und ich habe dadurch, wie ein Angeklagter vor dem Gericht nichts zu verlieren, alle Möglichkeiten der Freiheit.

Das ist, ich sehe mich nicht als Angeklagter, der jetzt lügen muss, um nicht verurteilt zu werden, sondern ich sehe in diesem Angeklagten die Position die bis zum Letzten nach der Existenz durchleuchtet ist, bis zum letzten mit einer Artikularität darüber zu denken und so zu handeln. 
Ich glaube nicht, dass Künstler bessere Menschen sind, auch wenn sie ehrlich sind und sein wollen bei der Arbeit. Das beschränkt sich in erster Linie auf die Arbeit. Natiirlich hat dieses radikale Ehrlichsein, das man anwendet, gewisse Folgen. Aber ich glaube nicht, dass der van Gogh z. B. von der durchschnittlichen Bevölkerungsgruppe als besserer Mensch gesehen wurde. Wenn er heute wieder so leben würde, würde er genauso ausgelacht. Der war nur verriickt. Man stelle sich vor, er hat sich vor 100 Jahren an einen Hut Kerzenleuchter hinauf getan, um in der Nacht besser malen zu können und die Staffelei mit Eisenstangen in den Boden gerammt, damals wurde das von niemandem gemacht. Es konnte aber damals auch trotzdem eher umgesetzt werden, das war noch leichter möglich als jetzt. Heute kann man in großen Städten verrückt sein - ich habe kein Bedürfnis zu Verrücktheit in dem Sinne, aber ich spüre im Magen, dass ich eben anders bin und das ich anders reagiere auf gewisse Dinge und das würde jemandem in einer Großstadt wie New York weniger auffallen. Hier in Graz fällt das natürlich extremst auf, muss man sagen. Es fällt schon am Land wieder weniger auf als in Graz oder wo Du herkommst. In Tübingen wird es ziemlich auffallen, wenn man ein verriicktes Leben führt.

SR: Ja, Tübingen ist ziemlich spießig.

GR: Ja, genau, wenn man ein Leben führt, das komplett gegen den allgemeinen Raster geht. Ich habe jetzt das Stipendium ein Jahr in Hamburg gehabt. ${ }^{20}$ Es war mir unmöglich

${ }^{20}$ Im März 1979 wurde Roth vom Hamburger Kultursenat ein neunmonatiges Stipendium des Förderungsprogramms Auswärtige Künstler zu 
dort mein Leben zu leben. Es war unmöglich. An der Haustür war eine Glocke ...

SR: Das war Dir ein Leiden, das Jahr in Hamburg?

GR: Das war es richtig. Aber ich möchte alles wissen im Leben, mir ist das Leiden wurscht. Ich nehme das Leiden genauso an, mir ist es egal. Es war mir ja so interessant auf diese Weise. Es gibt nichts im Leben, bis auf den Tod der Kinder, was mir Schrecken einjagen kann. Der Tod der Kinder wäre wirklich das Einzige, wovor ich mich fürchten würde, aber ansonsten ...

Ja, da in Hamburg, da war es so, das war eine ebenerdige Wohnung, die erste neben der Tür und eine Berufsschule war gegenüber und da haben mir die Kinder manchmal angeläutet, um sieben Uhr, halb acht und sind weitergelaufen, oder am Abend, irgendwelche Besoffene haben bei der Türglocke angeläutet und ich musste jedes Mal am Tage, wenn ich geschlafen habe, musste ich jedes Mal aufstehen. Die Handwerker, wenn sie kamen, haben immer bei der ersten Glocke angeläutet, das war ich. Die Post, wenn sie gekommen ist, hat auch immer angeläutet. Es waren keine Briefschlitze. Auch um Pakete abzugeben, dann war der Nachbar nicht da, wenn ihn jemand gesucht hat. Wenn etwas war, war ich die erste Glocke, nicht dass man vielleicht den Hausmeister geholt hat, man hat bei mir angeläutet, weil ich eben die erste Glocke war. Das war so eingerichtet, dass das Telefon nicht neben dem Bett war und ich musste da jedes Mal aufstehen. Natürlich telefoniert die Menschheit schon

Gast in Hamburg verliehen. Roth erhielt eine kostenfreie möblierte Wohnung und DM 2.000 pro Monat. 
am Vormittag. Ich habe trotzdem in der Nacht nicht schlafen können, so musste ich am Tag schlafen und da waren jedes Mal um zehn Unterbrechungen. Dann die ersten sechs oder sieben Wochen, die ich draußen im Herbst war, das war sehr arg, weil da habe ich überhaupt niemanden gekannt und ich wollte nicht immer nur Kunstleute kennen lernen, sondern ich wollte leben. Ich wollte keine anderen Schriftsteller treffen, was ich später dann gemacht habe, aber nicht so, dass ich da lästig werde, weil ich niemanden hatte, sondern erst, wenn ein gewisses Umfeld gegeben war.

Und da war der einzige Gesprächspartner von mir, den ich am Tag gehabt habe, der Herr Buchhändler Wohlers in der Langen Reihe mit einem wunderschönen Antiquariat. Der wusste natïrlich auch nicht, was los ist, nicht. Ich bin in der Frühe aufgestanden und am Abend hingegangen und der einzige Gesprächspartner war er. Ich habe das Essen in Lokalen dann aufgegeben, habe nur immer am Stand gegessen, weil mir nicht lieb war, immer dort zu sitzen mit den Zeitungen alleine an einem Tisch. Das hat mir nicht gefallen und ich war auch so... Ich bin herumgegangen tagelang oft den ganzen Hafen entlang, in den Friedhof, ich bin um die Alster herum, im Winter über die Alster drüber, ich bin wie besessen gegangen dort. Aber ich hatte immer eine Phase, wo ich kommunikativ schlecht war, wahrscheinlich, weil ich irgendwo die Kommunikation angestrebt habe, weil ich isoliert war und es mir aufgenötigt war, nicht freiwillig war und ich immer so den Gedanken hatte, es wird schon irgendwie gehen. Ich bin vom Land gekommen, wo ich jedes Haus gekannt habe und überall hineingegangen bin, es war für mich eine enorme Umstellung. Allerdings dann am Ende, als ich weggegangen bin, habe ich viele, viele Menschen kennen 
gelernt und es war sehr schön und abwechslungsreich. Diese eine Phase war eben ziemlich schwierig zu bewältigen. Ich habe in erster Linie Trinken und Schlafmittel benötigt. Schlafmittel waren notwendig, ich habe ja tagelang, zwei oder drei Tage nicht geschlafen oder ich bin um zwei in der Fruihe aufgestanden und vor meinem Haus hat es eine Bar gegeben, so ungefähr fünf Minuten zu Fuß, die hat „Endstation" geheißen. Die hat vierundzwanzig Stunden offen gehabt und ich bin um zwei Uhr früh bis sieben Uhr, bis es hell war, trinken gegangen. Da haben sie mich schon gut gekannt, Zuhälter usw. - im selben Haus ist das Hotel Bristol, ein Bordell. Mit den entsprechenden Damen habe ich mich schon sehr gut unterhalten, versorgt mit Aalen und allem Möglichen. Die wussten natürlich nicht, wie es mir geht oder vielleicht haben sie das sehr wohl gewusst und ich glaubte nur, sie wussten es nicht. Aber dann später bin ich dort auch wieder hingegangen, da war es dann eher als Spaß - wirklich aus guter Laune mit Freunden und Bekannten. Während das am Anfang für mich eher eine Notwendigkeit war, nicht. Diese Stunden zwischen zwölf und vier Uhr, da sitzt ja kein Mensch mehr in einer Bar, man sitzt allein mit dem Barkeeper dort und ein zweiter Besoffener hängt da, es geht nichts mehr vorbei - eine seltsame Zeit.

SR: Eine Hölle.

GR: Ja, es war eine neue Erfahrung und wie ich es jetzt sehe, bin ich froh, dass ich in dieser Form und nicht anders alles selbst erlebt habe. Mir passt es so, mir ist es existentiell recht und ich möchte nicht in einem Literaturbetrieb drinnen sein, der mir das wegnimmt. Ich mache z. B. seit zwei oder 
drei Jahren überhaupt keine öffentlichen Lesungen. Es hat zwei Gründe. Der erste ist, dass ich mich irgendwie unwohl fühle bei einer Lesung.

Und zweitens lernt man da ein Leben vorzugaukeln, das mit dem wirklichen Leben nichts zu tun hat, das ist alle Humbug. Ich kenne viele Freunde, die das lieben, weil sie sich dort bestätigt fühlen oder es ist ein gewisses erotisches Sprungbrett so abends zu lesen immer, das ja nicht unangenehm ist, aber ich glaube, dass es besser ist für mich - es muss das jeder so machen, wie er das für richtig hält - dass ich mich aussetze irgendwie und zwar bewusst nichts gewaltsam dazu tue, um meine Situation, in der ich stecke, zu ändern. Also, ich suche keine schlechte Situation, ich bin nicht nach Hamburg gegangen, um dort vor die Hunde zu gehen oder Säufer zu werden oder zu verzweifeln sondern eigentlich, weil mich Hamburg interessierte und ich wollte gern einmal dort sein. Es hat mir gut gefallen. Das ist von selber gegangen.

SR: Ich war selber vor zwei Wochen in Hamburg - unterwegs nach Norden...

GR: Aber der Winter, der Winter ist furchtbar, nicht.

SR: Die Stadt gefällt mir sehr.

GR: Ja, die Stadt ist gut, sie ist eine Arbeiterstadt...

SR: Ich bin selber in einer Hafenstadt aufgewachsen. 
GR: Hamburg ist auch eine Arbeiterstadt. Es ist fast überall gut, wo eine Arbeiterstadt ist und in solchen Gebieten ist es immer großzügig. Da ist das Land, wo ich bin, viel kleinlicher im Vergleich. Nur das macht mir nichts aus. Das Land hat wieder andere Vorteile, z. B. dort am Lande, wo ich bin, ist der konservative Teil, der mir angenehmere Teil - im Aufenthalt. Die Arbeiter konnten ja keine Kultur dort entwickeln, nirgendwo, es gibt keine Arbeiterkunst in dem Sinne, wie es eine Bauernkunst gegeben hat, wo das Haus aus sich selber heraus, die Möbel aus einer Notwendigkeit gekommen sind und auch noch bewusst geformt worden sind, von den Gegenständen bis zum Haus selber, die Werkzeuge und das Gewand, alles war aus sich selber heraus gespeist. Der Arbeiter am Land, wie ich ihn kennen gelernt habe und ich kenne viele Arbeiter, mein Großvater war Glasmacher und als Kind bin ich mit ihm mit den Arbeitern zusammen gewesen. Ich bin sehr gerne unter Arbeitern. Aber dort am Land sind sie halb Bauern und halb Arbeiter; sie gehen in die Stadt arbeiten und leben aber am Land und das hat eine seltsame und irgendwie lustige Mischung hervorgebracht. Sie sind so strebsam und nett und anständig, das ist aber langweilig irgendwo. Die richtigen konservativen Bauern sind ja Lügner alle, nicht. Kein Städter hat da überhaupt eine Chance bei einem Bauern. Wenn Du nur dreimal ,ja“" sagst zu etwas, bist Du schon für 20 Jahre verkauft. Sie sind einem enorm überlegen. Bei aller Liebe zu den Menschen, die dort leben, muss man einfach sagen, muss man auf der Hut sein bei ihnen, weil sie einen im nächsten Augenblick hineinlegen. Das betrifft das Gut, das betrifft, was man ihnen abkauft. Es ist interessant, die meisten Städter kommen immer mit einem schlechten Gewissen aufs Land. Die schen- 
ken dann den Leuten dort Gewand, die Wochenendler, nicht. Die Bauern, die lachen uiber jeden insgeheim. Sie nützen es aus, sie nützen alles aus wie sie den Boden ausnützen und den Grund ausnützen und ihre Körperkraft bis zum letzten ausnützen. So nützen die jeden anderen, wenn die Möglichkeit besteht, aus. Das ist in ihrem Weltplan drinnen. Der Bauer hat zu allem ein geschäftliches Verhältnis, weil seine Existenz gleichzeitig sein Geschäft ist. Der Boden, der Mais, das ist alles Materie und genauso die Umwelt. Wenn ich dort hinkomme, bin ich natülich gern gesehen, wenn ich Geld eingesteckt habe und Zeche mache. Aber ich habe das gern. Es kann einen natürlich ärgern, aber ich sehe das ganze wie ein Spiel an.

SR: Bei den Erzählfiguren in Deinen Romanen ist ein Verwandlungsprozess $\mathrm{zu}$ spüren: einstein wird Kalb wird Künstel wird Haid wird Weininger wird Nagl usw. Es liest sich wie ein langes Filmwerk.

GR: Ja, es ist möglich, dass es so ist. Ich habe es selbst nicht beabsichtigt. Es ist nicht so, dass ich immer dieselbe Figur in dem Sinne beschreiben will, aber schließlich und endlich bin ich immer irgendwo ich. Und nicht, weil ich mir selbst so wichtig bin, nicht weil meine Existenz mir so wichtig erscheint, sondern weil ich mich so gut kenne oder zumindest dabei bin, mich täglich gut kennen zu lernen und auf Neues draufzukommen.

SR: Vielleicht ist das so, weil das Selbst ein ungeheures Ding ist mit unendlichen Möglichkeiten. 
GR: Eben, das ist überraschend eigentlich, nicht, dass man selbst so viele Möglichkeiten in sich findet, ist eine anregende Sache. Es ist gar nicht so, dass ich selber der Ausgangspunkt bin, dass ich denke, ich schreibe das jetzt über mich oder das bin ich, sondern dass ich eine Figur tatsächlich jedes Mal erfinde und dann daraufkomme, im Nachhinein, dass ich es bin. Während ich schreibe bin ich der Meinung, dass es alles Erfindung ist, und ich gebe von mir Ansichten dazu, damit das schön wahrheitsmäßig ist. Unabsichtlich gebe ich aber auch vieles andere von mir her und im Grunde genommen bin ich froh, dass es so ist, weil ich irgendwie so ein Gefuihl habe auf einer Spur zu sein, auch wenn es einem nicht passt, auch wenn es einem oft betrügt, aber ich akzeptiere schon für mich als Glück, dass ich Spuren machen kann und für mich war es so, dass ich glaube, je länger man schreibt, desto mehr verliert man die Unverschämtheit, die am Anfang beim Schreiben da ist. Am Anfang beim Schreiben da ist, ja eben fast wie eine Form von Hochmut; es ist keine Form von Bescheidenheit, sondern es ist eine Form von Arroganz irgendwo am Anfang gewesen. Dass man es so wichtig findet, dass man selbst was aufschreibt und diese Fiktion, dass man es nur für sich alleine macht, die kann ja sicher nicht halten. Das konnte man als Kind machen als man eigene Bücher gemacht hat, aber das lässt sich nicht aufrecht erhalten. Den reinen Künstler, den sich die Gesellschaft wünscht, den gibt es nicht. Man kann sich natürlich das Layout eines reinen Künstlers geben, auch der reine Künstler ist in einer Weise berechnend, dass er immer Publikum will. Der Kleist hat sich aus Ehrgeiz umgebracht; ich glaube auch irgendwo der van Gogh aus Verzweiflung, die mit dem Ehrgeiz zusammenhängt. Bei mir 
wird das Bewusstsein des Scheiterns eigentlich immer deutlicher - dass man nicht erreicht in einem Buch, was man erreichen will, dass man nicht diese ... im Kopf ist es besser.

SR: Ist es Dir eigentlich möglich die Welt zu betrachten ohne sie zu beschreiben?

GR: Wir haben etwas Ähnliches heute schon besprochen: es ist irgendwo auch das Gluick es zu können, auch es tun zu dürfen. „Dürfen“ sage ich nach einer sehr schweren Krise heuer im Sommer; ich habe ja von Juli bis September die schwerste Krise gehabt, die ich je zu überwinden hatte. Ich habe mir nur gewünscht, dass diese Leere da in mir, so ein bisserl ein Loch im Kopf, dass das verschwindet. Ich habe auch was ich bisher geschrieben habe, wie etwas von einem Fremden betrachtet, als hätte ich damals ein anderes Leben gefuihrt und jetzt war ich auf einmal, ja, eben, wie ich es am Anfang ausgedrückt habe, Selbstmordkandidat. Alles geht dann nur mehr um das Momentane für mich, in dem Moment, wo diese Dimension des Schweigens weg ist nur wieder Moment - natürlich in der Liebe oder in vielem, im Fressen, Saufen, ich denke keine Minute an das Schreiben, obwohl es immer in mir drinnen ist. Aber es ist wie ein Fluch, zuerst der Wunsch es zu verlieren, dann kommt es wieder zurïck - da ist eben nicht so eine Kontinuität. Natürlich es gibt das auch - Momente wo man nur schaut und glücklich ist mit dem Schauen, aber das ist dann so stark, dass wenn man schreibt, man sich gerade an das erinnern kann, weil es gerade in dem Augenblick so stark war. Dann ist es keine Normalität. Ich kann mir vorstellen, dass ich eine zeitlang oder gar jahrelang etwas anderes mache. Also 
zum Beispiel, so wie ich jetzt eben am Land herum gegangen bin oder Taxi fahren. Taxi fahren überhaupt würde mich ein bisserl interessieren - das ganze Menschentheater, das für den Taxidriver zu sehen ist - das wäre schon als existentielle Situation interessant. Ich habe aber keinen Führerschein.

SR: Kann es sein, dass das Schreiben Deine Art ist, das Leben zu lieben? - ein Ausdruck dieser Liebe.

GR: Ich gehe nicht hin, um aus Liebe zu schreiben, sondern ich kann im Schreiben schon auch lieben, aber es ist nicht, um das auszudrücken. Ich würde es auch als Instrument um meinen Hass auszudrücken benutzen oder mein Glück auszudrücken oder meine Verzweiflung oder was auch immer auszudrïcken. In irgendeiner Weise ist es Selbstzweck und das ist das, was auch die Leute ärgert. Es ist eine Art eigener Natur so irgendwie, Selbstzweck ohne dass da jetzt für mich Absichten dahinterstecken. Ich könnte mir nie vorstellen, ich setze mich jetzt hin und schreibe einen Roman, von dem ich 50.000 Auflage habe oder ich könnte mir nicht vorstellen, ich schreibe ein Stück, das von 10 Bühnen gespielt würde, sondern das ist ein Thema, das mich interessiert oder mich fasziniert und ich verwickle den Leser, den imaginären Leser, in ein Spiel und möchte, dass er mir folgt, möchte ihn überraschen und möchte ihn orientalische Düfte bescheren und plötzlich die Tür geht auf und das nächste Mal möchte ich ihm ein Lichtspiel zeigen oder ich möchte ihn verblüffen oder ich möchte ihn an der Nase herumführen - ich spiele mit ihm. Der Leser, dem ich das vorfuihre, dem gilt mein ganzes Interesse und ich teile sozusagen das was ich an Lie- 
be empfinde in den Figuren, die ich schreibe und in der Vorstellung des imaginären Lesers auf. Nach Ende eines Stückes oder einer Prosa möchte ich das ausprobieren, wie das aufgegangen ist. Und das ist der ganze Prozess. Wenn es bei vielen aufgeht, dann hat man irgendwie den Eindruck, dass das eigene Konzept möglicherweise aufgegangen ist. Wenn es bei wenigen aufgeht, dann hat man den Eindruck, dass die Menschheit noch zu dumm ist, um es zu verstehen: das ist die einzige Möglichkeit mit diesen beiden Alternativen zu überleben. Das wäre, glaube ich, die falsche Form eines Künstlertums. Es sind ja auch die Dichter oder die Maler, die nicht zählen, die so vorgehen können, dass sie sagen: „Ja! Da war es zu wenig Dings, jetzt muss ich meinen Stil ändern, probieren wir es auf diese Weise." Der Mann - oder die Frau - an den ich denke, kann das nicht anders machen als er das macht. Der ist nicht in der Lage. Ich kann z. B. auch gar keine Stilformen von Autoren länger als eine oder zwei Seiten kopieren, weil mich das krank macht. Ich könnte als Spaß etwas zitieren aus dem Hemingway oder Hamsun oder so etwas machen, kurz für mich. Also ich könnte es rein formal ausprobieren. Da kann ich einen Thomas Bernhardschen Schreibstil sofort nachahmen, aber es würde mich schon nach einer oder anderthalb Seiten maßlos ermüden, weil mit dieser Imitation sozusagen erstens die ganze Aufmerksamkeit auf die Imitation gerichtet werden muss und nicht auf die Figuren und das was sich abspielt. Und das ist eine beträchtliche Kraft, die verlorengeht oder, zweitens würde ich gar keine eigene Stimme haben, wenn ich das tu... 
SR: Das Wort „Stimme“ finde ich hier sehr wichtig. Ein Autor hat seine erzählerische Stimme. Du kannst sehr genau die eigene Stimme spüren beim Schreiben.

GR: Es gibt natürlich Ähnlichkeiten, das ist klar. Es gibt Wahlverwandtschaften, Ähnlichkeiten. Es ist ganz klar, dass man sagen kann: ,Na, gut...' Die Literatur ist ja auch keine Erfindung aus sich selbst heraus in dem Sinne. Die Technik zeigt es ja vor. Der Japaner, der diesen Apparat, den Sanyo [zeigt auf den Apparat] baut, da ist eben eine Geschichte von technischen Entwicklungen drinnen in dem Apparat, die immer in so einem Gerät mündet, und er hat ja nicht alles, was da drin ist, selbst von vorne allein erfunden. Der Lautsprecher sieht aus wie bei einem Braun oder so etwas, der Griff, der ist ja allgemein und der Empfänger ist ja allgemein da drinnen, aber das Ganze, so wie es jetzt da ist, ist dann ein Sanyo Gerät. Der Brecht hat gesagt: „Ich habe vieles entlehnt, nichts gestohlen!“ Und das ist irgendwo für mich das, was man sagen kann. Dass man ganz unbeeinflusst bleibt von Autoren, die wichtig sind, von Italo Svevo, z. B., den ich jetzt noch dazurechnen möchte mit seiner Cousine. Es gibt keinen Autor, der gar nicht begeistert ist von anderen Autoren. Über die ist man ja zur Literatur gekommen. Es muss halt dann gefiltert sein durch die eigene Person, dass man sagen kann, das kann nur der machen oder es hat nur der machen können. Wenn etwas überhaupt übrig bleibt vom Leben eines Schriftstellers, dann ist es das, was nur er machen konnte, das, was für ihn typisch war. Das ist halt jetzt schwerer als es früher war - jetzt haben wir die Bücherschwemme und die Vielfalt der ganzen Medien. Ich glaube nicht, dass Vieles, das heute so groß ist, noch in zehn 
oder fünfzehn Jahren so groß sein wird. Es ist mir ganz klar, dass dieses Bild sich verändern wird. Es bestimmt in Deutschland eine Handvoll, eine Gruppe von Kritikern was gut oder schlecht ist. Ich habe das Gliick momentan, dass ich nicht übersehen werde, dass ich irgendwie registriert werde, aber es ist eine furchtbare Abhängigkeit von einer Kritikergruppe in Deutschland. Österreich hat gar keine Zeitungen, kann da gar nicht mit. Uns fehlen sowohl Kritiker als auch gute Tageszeitungen. Die Kritiken in einer österreichischen Zeitung zählen nicht. Wir haben keine einzige wichtige Tageszeitung in Österreich, keine einzige gute Tageszeitung. Es ist auch die Misere warum sich die österreichische Literatur in Deutschland abspielt, das ist einer der Hauptgründe dafür.

Außerdem glaube ich, dass ohnedies das Scheitern bei mir auch irgendwann einmal kommen muss. Ich kann mich halt nicht zwangsläufig mit der Zeit bewegen und dauernd anpassen, sondern es wird dann eben so kommen. Ich mache mich jedenfalls darauf gefasst, das gehört dazu. Im Grunde genommen möchte ich es auch einmal erfahren.

\section{$* * *$}

GR: Wir haben gesprochen über den Zusammenhang von Verzweiflung und Geschlechtsverkehr, von Schmerz und Sexualität, meine Erfahrung ist, dass man in der Situation mutiger ist vielleicht was alle geschlechtlichen Dinge betrifft, wobei es aber keinen Mut mehr gibt in der Verzweiflung, weil ja kein Mut mehr notwendig ist, aber von außen betrachtet würde man sagen mutig. Ich glaube auch, dass die Erfüllung damit nicht verbunden ist, es ist ein Rausch, und 
zwar ein Gefühlsrausch, der aber nicht so stark ist, dass man nicht alles klar sehen würde. Das ist das Seltsame. Es gibt da beim Sartre eine schöne Geschichte die heißt Herostrat, wo ein junger Bursche sich mit einer Pistole in ein Klosett einschließt und einen Amoklauf unternehmen will und er geht mit einer Hure aufs Zimmer. Der hat diese Situation ziemlich deutlich und genau beschrieben in dieser einen Erzählung. Ich wollte, wie gesagt, dem nicht ausweichen und habe dann versucht, es in richtigen Abständen zu beschreiben. Und jetzt ist natuirlich die Frage gewesen, jetzt ist das drinnen und wie geht das ganze Buch jetzt weiter mit dem Geschlechtsverkehr? Ich kann ja nicht auf Seite 25 einen Geschlechtsverkehr machen und das ganze restliche Buch dann sagen, mit der Verzweiflung denkt Euch den Rest jetzt dazu. Da muss man sozusagen bei der Sache bleiben. Ich musste dieses Vorhaben bis zum Schluss durchhalten. Ich würde sagen, das war für mich eher ein mathematisch-literarisches Problem, die Häufigkeit, der Struktur nach, als ich es als Programm wissen will. Es ist für mich überhaupt kein Programm, sondern das ist eine Methode etwas zu zeigen und innerhalb dessen, was ich schreibe, dem den Platz zuzuweisen, von dem ich glaube, dass er ihm innerhalb meiner Arbeit gebiihrt.

SR: Ich habe Winterreise letzte Woche wieder gelesen. Es ist genau die Szene, in der man sich erkennen kann und in der ich mich auch erkennen kann.

GR: Ja für mich war das Erkennen von mir selber in den Büchern immer Trost. Das war für mich immer schön, dass jemand anderer das so gedacht und gefunden hat und auch 
so mutig war, das zu schreiben, dass das so genau und wahrhaftig war, das hat mir oft Kraft gegeben und ich verstehe das Schreiben auch so, dass ich für die schreibe, die diese „Helden“" - unter Anführungszeichen - notwendig haben zur Selbsterkennung, wie ich es mache. Mein Leser, wie ich ihn mir heute vorstelle, ist einer, der zumindest Probleme hat, würde ich sagen.

SR: Wenn ich so etwas lese, habe ich das Gefühl, dass ich wieder ein Mensch bin, dass diese Helden meines Lebens wirklich existieren. Ich bin nicht der Einzige, der solche Erfahrungen gemacht hat. Das ist eine sehr wichtige Funktion des Schriftstellers.

GR: Das ist das Einzige, was ich wirklich beabsichtige. Neben diesem Spaß eben zu schreiben und den Leser an der Nase herumzuführen - ich habe das einmal im einstein geschrieben, diese zehn Punkte da. Daneben habe ich immer das Bedürfnis mich an einen Einzelnen, der Schwierigkeiten hat, zu wenden. Das ist auch der Leser, den ich gerne habe und mit dem ich mich unterhalte und ihn auch verstehe. Und es sind auch meistens solche Menschen.

SR: Auf der anderen Seite quält es mich, das zu lesen.

GR: Es muss natürlich quälend sein, das ist ja klar. Wenn mir jemand sagt wenn ich krank bin, was ich wirklich habe, quält es mich und trotzdem bin ich zufrieden, dass ich weiß, was los ist und weiß, es gibt auch andere, die haben das auch und wie die damit fertig werden. 
SR: Es kommt ein anderer Satz in Winterreise vor: „Er hatte das Gefühl als sei er aus der Erde gefallen. Es war ein ozeanisches Gefühl der Einsamkeit.“ Das Wort „ozeanisch“ hier?

GR: Kommt auch als Anspielung auf Freuds „Das Unbehagen in der Kultur" - da spricht er von einem ozeanischen Gefühl. Das ist ein kurzer Aufsatz, sehr gut und interessant. $^{21}$

SR: Eine andere Idee im Roman, die mich da ergreift: „Es schien Nagl als sei es das Normalste, sich selbst auf-

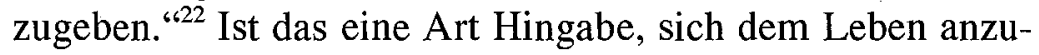
vertrauen?

GR: Die Verführung, sich selbst aufzugeben, ist ja immer da. In dem Moment, wo man sich aufgibt, lebt es sich leichter. Es ist oft sehr schwer, die eigene Meinung zu vertreten und man muss oft unterscheiden, ob das jetzt Eitelkeit ist oder ob das für einen persönlich von Wichtigkeit ist. Man kann es sich ja sehr leicht machen, indem man immer auf seinem Standpunkt beharrt und nie nachgibt. Das würde ich nicht als günstige charakterliche Veranlagung sehen. Die Schwierigkeit besteht darin, die Punkte aus einem zusammenhanglosen Alltag herauszufinden, die für die eigene Person in der Entscheidung von Wichtigkeit sind. Man verhält sich ja oft in einer Art Besinnungslosigkeit, dass man nervös ist, mit jemand unfreundlich ist, aus irgendwelchen mechanischen, nervösen Gründen. Man lebt ja viele Verhal-

${ }^{21}$ Sigmund Freud ,Das Unbehagen in der Kultur.' Abriß der Psychoanalyse/Das Unbehagen in der Kultur, Frankfurt/M., 1972.

${ }^{22}$ Winterreise, S. 5. 
tensweisen in einer Art Besinnungslosigkeit durch. Und sich selbst zu behaupten, heißt ja, aufmerksam zu sein, dass man nicht in diese Besinnungslosigkeit verfällt, sondern ich gebe mir nicht automatisch recht, ich gebe mir recht, nachdem ich es mir überlegt habe. Es mag sein, dass das im Alltag dann gar nicht mehr auffällt, dass das so eine Reaktion ist, wie sie jeder hat. Sich selbst aufgeben, bedeutet für mich, das zu machen, was die anderen machen. Das ist also nicht der Selbstmord für mich, sondern es ist die Selbstbehauptung des Selbstmordes.

\section{SR: Selbstverlust?}

GR: Eher so, ja. Das es sozusagen normal wäre. Viele Menschen, wenn sie in der Pubertät sind - die meisten eigentlich - haben eine künstlerische Begabung, etwas Außergewöhnliches an sich in der Pubertät. Und schnell ändert sich das und die Menschen werden wieder grau und eintönig, wenn sie älter werden, mit 20 oder so. Und nur wenige, sozusagen "Wahnsinnige" behalten sich diese Kraft aus der Pubertät, die bleibt ihnen. Es ist fast jeder Künstler pubertär. Er könnte gar nicht anders sein. Er muss Freude an Spielsachen haben. Die Umwelt und alles sind ja alles irgendwo wie Spielsachen. Wie ein Auto über die Straße fährt, die Einstellung eines Kameramannes, dass er ein schönes Bild macht, das ist alles ein Spielzeug, das man sich selber macht. Und die Aufgabe der eigenen Person bedeutet dann, dass behauptet wird, das sei eben die Wirklichkeit, das sei das Leben, es gäbe eben nichts anderes und da gibt es dann in meinen Augen wieder eine Menge so genannter Verrückter, die unter diese Gruppe zu finden sind: Kinonarren, Jazznarren, Fuß- 
ballnarren, die sich in ihrem Leben eine zweite Wirklichkeit bauen, um diesen jugendlichen, Wahnsinn' zu behalten. Dass dann doch noch irgendwo ein Traum da ist, der das Leben miterfüllt. Ich habe eine Geschichte übers Fußballspielen geschrieben Roth schlägt Leeds United, bin tausende Male auf Fußballplätzen gewesen und ich kenne den so genannten Fan, für den ist das eine Bereicherung seines Alltags, der hat dann für die ganze Woche Gespräche mit Arbeitskollegen und man wartet dann schon auf das nächste Spiel. Sich selbst aufgeben heißt dann, sich mit so einem Hobby zufrieden zu geben und das zum zweiten Inhalt seines Lebens zu machen, das wird dann eine Art Ersatzreligion und alles, was in einem ist, meldet sich halt dann trotzdem. Dann kommt das Saufen usw. dazu. Das verstehe ich also unter sich selbst aufgeben. Der sich selbst behaupten will, für den gibt es eigentlich immer nur schwere Tage, der ist für jeden Tag, der heiter ist, dankbar.

SR: Und Nagls Entschluss am Ende des Romans mit dem Flugzeug nach Alaska au fliehen?

GR: Ich habe einmal einen Film mit dem Humphrey Bogart gesehen. Da ist er mit seiner Freundin auf eine Alm gegangen und vor der Polizei in den Schnee geflohen. Einen zweiten Film habe ich gesehen Liebende Frauen von Ken Russell, das D. H. Lawrence-Buch. Da geht auch einer in der Schweiz auf einen Berg hinauf und wie ich das damals gesehen habe, habe ich mir gedacht, wenn ich das nächste Mal eine Gelegenheit habe, irgendwie in einem Buch das unterzubringen, dann möchte ich das einmal beschreiben oder anwenden. 
SR: Ein Freund von mir hat genau das gemacht, 1971.

GR: Und was hat er einige Monate dort gemacht? Ist er auch verschollen?

SR: Er hat dort gearbeitet, er hat sich nach ein paar Wochen eine Stelle gefunden.

GR: Und was macht er jetzt?

SR: Er ist Lehrer, in Neuseeland wie die anderen in seiner Familie.

GR: Na ja, man muss ja nicht droben bleiben. Grönland wäre für mich zu pathetisch gewesen, ich wollte etwas finden, was Kälte ausdrückt. Es sollte ein Satz das abschließen und nicht ausgeführt werden, was er macht. Ich verabschiede mich von meinen Figuren kaum in Aussichtslosigkeit, ich mag sie nicht so zurïcklassen, dass überhaupt keine Aussicht mehr besteht. Das war beim Großen Horizont so, das war beim Neuen Morgen so, das ist auch bei der Winterreise so. Da erspar ich mir sozusagen ihn zu richten und ich habe z. B. die ganze Zeit ein schlechtes Gefühl, dass ich den Ascher Selbstmord machen lasse im nächsten Buch, weil er mir leid tut. Er tut mir richtig leid, aber es muss sein, da kann man nichts machen.

SR: Ich finde das Melville Zitat zu Beginn des Stillen Ozeans wieder bedeutsam: „Jetzt, da alles mit Schnee bedeckt ist, habe ich hier auf dem Lande das Gefühl als wäre ich auf See." Das bezieht sich auf die Winterreise, nicht? 
GR: Ja sicher, aber es ist so gedacht - ich mache schon immer Anspielungen auf die Bücher vorher, es hängt ein Buch mit den anderen zusammen. Es gibt keine Buicher, die leer in der Luft stehen, mir hat das immer sehr gut gefallen, wenn ein Autor so Personen durchgezogen hat, wie bei Hemingway so eine Art Biographie von A bis $Z$ oder von Miller und der K. bei Kafka. Irgendwie müssen die Bücher alle zusammenhängen, das ist so ein Gefühl von mir, dass das so sein soll. Natürlich komme ich nicht sofort von einem Thema los und bin schon beim nächsten, und von einer Sichtweise und von einem Gedanken, sondern das ist alles noch da, das ist ein unerledigter Rest in mir, das was nicht aufgegangen ist, geht weiter. Und das Melville-Zitat habe ich eigentlich so als Klammer zwischen dem Titel des Romans und dem Beginn, dass also gleich die Einstellung da ist. Der stille Ozean - da weiß man erst einmal nicht, was damit gemeint ist und durch die kurze Information des Melville-Zitats, das aber gleichzeitig mit Ozean und mit Land zu tun hat, ist die Richtung des Buches bekanntgegeben. Man hat mir vorgeworfen, ein Kritiker in der Rundschau hat so Vergleiche gemacht zwischen Melville und mir und mir vorgeworfen, dass ich es mir leicht mache. ${ }^{23} \mathrm{Er}$ hat das Zitat angegriffen, was natürlich eine Dummheit ist, weil das Zitat nur die Funktion hat, zwischen dem Titel und dem Beginn der Geschichte zu vermitteln. Ich musste da einfach irgendetwas hineintun. Diese Klammer musste ich irgendwo anbringen zwischen

\footnotetext{
${ }^{23}$ W. Martin Lüdke, ,Jagdszenen in der Steiermark: Gerhard Roths neuer Roman Der stille Ozean', Frankfurter Rundschau, 5. Juli 1980: „Roths Roman, der in der südlichen Steiermark spielt, den ,stillen Ozean' allein im Titel trägt, will - vielleicht um der Verzweiflung zu entgehen - von Melvilles ungeheuerer Anstrengung zehren."
} 
dem Titel und dem Beginn. Da war das Buch schon fertig, ich hatte schon die erste Korrektur hinter mir, als ich das Zitat von Melville zufällig gefunden habe.

SR: Ich habe mich vor kurzem mit einem amerikanischen Dichter beschäftigt, für den Melville der wichtigste Schriftsteller ist.

GR: Wer ist das?

SR: Charles Olson

GR: Ja, den kenne ich, der war ja bei der Beatgeneration?

SR: Der Rector des Black Mountain College. Er hat über Melville geschrieben, zunächst eine Magisterarbeit. Danach hat er jahrelang weiter geforscht.

GR: Gibt es das als Buch?

SR: Ja, ja. Seine nicht eingereichte Havard Dissertation.

GR: Wie heißt das?

SR: Call me Ishmael. ${ }^{24}$

GR: Der Lüdke, in der Frankfurter Rundschau hat das Buch von Olson auch erwähnt. Es gibt ja auch einen Olsen in meinen Büchern, im Neuen Morgen, der Detektiv.

${ }^{24}$ Charles Olson, Call me Ishmael, San Francisco: City Lights Books, 1967. 
SR: Vielleicht jetzt etwas über das typisch Österreichische in Deinen Romanen.

GR: Zuerst muss man das etwas historisch sehen.

Das, was man in der modernen Literatur als typisch österreichisch bezeichnet, beginnt mit dem Zusammenbruch der Österreichischen Monarchie in der Folge dann eine Literatur, die weitgehend auf den Zusammenbruch der Monarchie Rücksicht nimmt, z. B. Joseph Roth, Musil; sie sind zwei Autoren, die ohne den Hintergrund der Monarchie nicht denkbar sind. Daneben gibt es den Prager Kreis, Hofmannsthal, den Sprachpolizisten Karl Kraus, auch der ist aus der Monarchie zu verstehen. Die letzten Tage der Menschheit z. B. Danach gab es einen zweiten großen Einschnitt in der österreichischen Literatur, den Nationalsozialismus, sodass die Generation, die nach 1945 geschrieben hat, in einem Staat gelebt hat, der geschichtlich für zwei Kriege verantwortlich gemacht wurde und beide Kriege verloren hatte. Aus dieser zweiten großen politischen Erschütterung hat sich auch in Österreich eine längere Zeit hindurch eine geschichtslose und formale Literatur gebildet. In erster Linie die Wiener Gruppe, ${ }^{25}$ die stärker vom Surrealismus oder Klebnikov, von Majakowski oder dem Dadaismus beeinflusst war als von sonst irgendeiner österreichischen Tradition. Die Wiener Gruppe mit Rühm, Artmann, Wiener, Achleitner, Bayer hat keine neue Literatur gegründet, aber sie

\footnotetext{
${ }^{25}$ Die Wiener Gruppe: Friedrich Achleitner, H. C. Artmann, Konrad Bayer, Gerhard Rühm, und Oswald Wiener.
} 
hat eine, für unsere spezifische Situation in Österreich wichtige und hier erstmals auftretende Form von Literatur eingeführt. Der Surrealismus ist beispielsweise in Deutschland weitgehend aus der Literatur herausgeblieben. Die deutsche Literatur ist daher relativ wenig vom Surrealismus beeinflusst worden. Dieser Einfluss ist in der österreichischen Literatur wesentlich stärker unter anderem deswegen, weil die Wiener Gruppe sich mit dieser Problematik intensiv auseinandergesetzt hat. Dazu kam die Entdeckung des Dialekts der Wiener Gruppe in den Mundartgedichten. Ich sehe in dieser Zeit den Ursprung der österreichischen Gegenwartsliteratur. Diese Beschäftigung mit der Literatur, wie sie z. B. Artmann, Bayer, Rühm, Wiener usw. gemacht haben, war in erster Linie eine intensive Auseinandersetzung mit der Sprache, zum Teil auf den Tractatus Logico-Philosophicus und die sprachlichen Untersuchungen von Wittgenstein zurückgeführt. Das heißt, es sind die geschichtlichen, politischen Ereignisse in der wichtigsten österreichischen Gegenwartsliteratur nach 1945 zuriickgestellt bis nicht beachtet worden. Diese Beschäftigung mit der Sprache, die zu ästhetisch hochwertigen Arbeiten geführt hat, hat auch gleichzeitig zu einer Reinigung der Sprache von einigen durch die politischen Ereignisse geschädigten Begriffen geführt. Es wurde wieder eine Dimension erarbeitet, in der man schriftstellerisch tätig sein konnte. Die Grazer Autoren sind nach der so genannten Wiener Gruppe um einen Schritt weiter gegangen, sie haben im Großen und Ganzen das, was die Wiener Gruppe an Sprache erarbeitet hat - an brauchbarer Autorensprache erarbeitet hat - wieder begonnen in eine neue Form des Erzählens umzusetzen. 
Ich teile nicht die Ansicht, dass diese Form des Erzählens, die jetzt in spezieller Weise von den Grazern gemacht wurde, etwas mit dem konventionellen Erzählen zu tun hat. Es hat in erster Linie etwas mit der Rückgewinnung von an Literatur Interessierten zur Literatur zu tun, und es ist der Neubeginn einer Beschreibung der österreichischen Umwelt und der österreichischen Mentalität. Langsam, über verschiedene Romane hat sich diese Form wieder entwickelt, z. B. die Romane von Peter Handke oder Romane von Alfred Kolleritsch, Stücke von Wolfgang Bauer, Romane von Barbara Frischmuth - sie sind aus einer neuen Sprache entstanden und haben sich wieder existentiellen und geschichtlichen Problemen zugewendet. ${ }^{26}$ Das spezifisch Österreichische an meiner Literatur ist also dieser Entwicklungsprozess, der von 1945 bis jetzt [1980] stattgefunden hat, d. h. die Beschäftigung mit sprachlichen Formen in fruiheren Arbeiten, mit experimenteller Literatur, meine Form der Sprachreinigung sozusagen und die Zuwendung zur Beschreibung meines Lebens in meiner Zeit, nachdem ich mir die sprachlichen Mitteln erarbeitet habe.

Darüber hinaus gibt es natürlich auch die andere Form des traditionellen Österreichischen, Der Mann ohne Eigenschaften, z. B. als Identitätsproblem, bei Hofmannsthal der Chandos-Brief oder die Verwandlung von Kafka ... Nun, nachdem diese Reinigung vollzogen wurde, nachdem diese geschichtlich-politische Barriere überwunden wurde, nachdem

26 Roth bezieht sich hier auf den Mitbegründer des Grazer Forums Stadtpark und Herausgeber der Literaturzeitschrift manuskripte Alfred Kolleritsch (geb. 1931) und auf prominente Vertreter der so genannten "Grazer Gruppe" der 60er Jahre: Peter Handke (geb. 1942), Wolfgang Bauer (1941-2005), und Barbara Frischmuth (geb. 1941). 
es eine Form von österreichischen Dadaismus gegeben hat man kann die Autoren der Wiener Gruppe vielleicht so nennen - reicht das Geäder der österreichischen Literatur wieder bis in die Gegenwart und schließt wieder an Themen, die in der österreichischen Literatur immer von Bedeutung waren. Man muss aber die Bedeutung von Franz Kafka z. B. für Handke, für Bernhard, aber auch für bestimmte Texte von Artmann erkennen. Ich würde sagen, dass Kafka der Autor war, der am unbeschadetsten von allen politischen Ereignissen hier in Österreich für andere Autoren wirksam war.

Wir haben in Österreich die geschichtlichen Ereignisse anders überwunden als das in der deutschen Literatur war. In der deutschen Literatur gab es eine sehr starke Auseinandersetzung mit dem Krieg, z. B. bei Böll und Koeppen, Die Blechtrommel von Grass, das fehlt bisher oder fehlte lange Zeit in der österreichischen Literatur und sie wendet sich langsam auch dieser Problematik zu - siehe Wunschloses Unglück von Peter Handke, siehe auch Die grüne Seite von Kolleritsch, Vor dem Ruhestande von Thomas Bernhard, Die Alpensaga von Turrini oder auch jetzt Passagen aus meinem eigenen Buch vom Stillen Ozean, wo es um die Auseinandersetzung mit der Geschichte geht. Was die österreichische Literatur dadurch an Stärke gewonnen hat, ist eine ästhetischere Form die Sprache zu behandeln, als sie in der deutschen Literatur zu finden ist, eine formalere und ästhetischere Form die Sprache zu behandeln. Was lange Zeit ein Verlust war, war das Außerachtlassen bis zu einem gewissen Grade der sozialen und gesellschaftlichen Bedingungen, die gerade geherrscht haben und herrschen. Es gibt aber eine Gruppe, die das politisch in Österreich ohnehin seit 
längerer Zeit macht. Dazu zähle ich z. B. Scharang, Innerhofer, Wolfgruber...

SR: Es kam Ende der Sechzigerjahre zu einer Auseinandersetzung zwischen Scharang und Kolleritsch über die Politisierung der Literatur, nicht wahr? ${ }^{27}$ Was war Deine Einstellung dazu?

GR: Meine Stellungnahme dazu ist, dass der Schriftsteller nicht der Handlanger irgendeiner Philosophie ist außer der persönlichen Philosophie des Autors. Ich empfinde mich nicht als Handlanger der marxistischen Ideologie und gar nicht als Handlanger der katholischen Ideologie oder sonst irgendeiner anderen.

SR: Was Handke z. B. in seinem Aufsatz Ich bin ein Bewohner des Elfenbeinturms zu der Frage der Politik geschrieben hat, ist das für Dich akzeptabel?

${ }^{27}$ Im Jahre 1969 verteidigte der Herausgeber Alfred Kolleritsch die so genannte "Theoriefeindlichkeit" der manuskripte gegen die kritischen Stimmen von u. a. Scharang und Elfriede Jelinek, die das westdeutsche Modell einer politisch-theoretisch engagierten Literatur befürworteten. Er behauptete, dass die kulturelle Maschinerie Österreichs wesentlich anders gestaltet wurde: ,In Österreich gibt es keine Kulturindustrie, die den Aufstand aufsaugte: hier gibt es nur ein Kulturdebakel, das endgültig würde, wenn man vor lauter unerfüllten Wünschen das Debakel in seiner historischen Zementiertheit selbständig wirtschaften ließe, ohne die lebende Kunst in den Bestand hineinzutreiben'. (Siehe manuskripte 25/1969, , marginalie'). 
GR: Nein. Das heißt, es ist für ihn akzeptabel: ich akzeptiere es so. Ich bin kein Bewohner des Elfenbeinturms. Ich setze mich auch mit den Ideologien auseinander. Eine Ideologie ist für mich ein Werkzeug und kein Käfig. Das ist der generelle Unterschied für mich zu anderen Autoren. Ein bestimmtes Werkzeug kann für bestimmte Reparaturen und Arbeiten notwendig, wichtig und vielleicht sogar ideal sein. Aber man kann nicht mit jedem Werkzeug alles reparieren, was es gibt. Jede Ideologie hat ihre begrenzte Anwendbarkeit. Der Marxismus kann nicht sämtliche Probleme des Menschlichen abdecken: dazu ist er nicht in der Lage. Er kann partiell den Wunsch nach einer sozialen Gerechtigkeit abdecken, aber nicht mein Gefühl der Trauer, der Freude, der Liebe usw. beeinflussen. Genau so ist es bei der Religion. Sie kann das Bedürfnis nach metaphysischen Projektionen abdecken. Der Autor darf sich nicht innerhalb einer Ideologie befinden wie in einem Käfig, in dem er also noch schaut, ob er jetzt wohl noch in der Ideologie ist, sondern ich glaube, dass der Autor die Ideologien kennen soll und sie dort anwenden, wo sie ihm etwas bringen, sich aber den Ideologien nicht ausliefern soll: Ich bin nicht so puritanisch in der Ansicht, dass ich in einem Elfenbeinturm leben muss, das mag ich nicht, ich setze mich selber aus. Für mich ist mein Leben auch gleichzeitig eine Form des Experiments, eine Form des Teilnehmens, Leidteilens, des Freudeteilens, aber immer mit dem Versuch zumindest Abstand zu halten und Distanz zu empfinden. Distanz zu empfinden zu dem, wie andere versuchen mir das, was ich sehe und erlebe, zu erklären. Ich möchte mir das erklären mit dem Wissen, das andere erarbeitet haben, dann doch selbst prüfen und versuchen, dem was ich schreibe, gerecht zu werden. 
SR: Was ist denn das Politische in Deinen Romanen?

GR: Ich glaube gerade beim Stillen Ozean ist sehr stark eine politische Idee drinnen, wenn man den Amoklauf des Lïscher am Schluss analysiert. Er begeht diesen dreifachen Mord aus dem erklärbaren Grund, dass er aus einer Winzerfamilie stammt, dass er in Abhängigkeit zu moralischen Gesetzen, zu einem aufgebauten politischen Regelmaß steht. So drückt der Stille Ozean sehr deutlich aus, dass in dieser Gesellschaftsordnung, in der ich diesen Roman spielen lasse, in der Füchse aus Tollwutverdacht erschossen werden, in der vieles mit Gewalt gelöst wird, da drücke ich schon eine Sehnsucht nach einem gerechteren sozialen Gefüge aus und fertige meine Analyse dafür an. Ich nehme dazu, glaube ich, sehr eindeutig Stellung.

In dem Roman Winterreise, da ist das Philosophische, Existentielle vor dem Politischen, d. h. hier geht es mir mehr um eine allgemeine philosophische Situation als um eine politische. Wenngleich es aber auch hier ein politisches Umfeld gibt, in dem man sagt, diese Form des Lebens gibt es in einem Staat, in dem es zumindest in keinem sehr überblickbaren Ausmaß wieder das Elend gibt. Es gibt noch eine andere Form von Elend als das soziale Elend.

Die amerikanischen Bücher enthalten beide bewusst Passagen mit gesellschaftspolitischen Auseinandersetzungen. Das Amerikabild, das ich meine Figuren erfahren lasse - und es auch mir so erschien, wie ich es erlebt habe - kann man ganz sicherlich ebenfalls als gesellschaftspolitisches Ergebnis bezeichnen, wenngleich mir jedes Mal nur diese Dimension zu wenig wäre. 
SR: Ja, ich erinnere mich an einige Stellen, wo im Großen Horizont der Haid Horkheimer zitiert. ${ }^{28}$

GR: Ja, bei Horkheimer gibt es Auseinandersetzungen mit der Armut in Amerika, mit der Frage nach der Gewalt usw. Es ist sehr subtil verstreut in den Texten. Aber ich glaube, dass von diesem Punkt an, von dem Großen Horizont an bis zur jetzigen Arbeit, die ja noch viel deutlicher dieses Element in sich tragen wird - Landläufiger Tod - eine kontinuierliche Kette von Auseinandersetzungen ist.

Die Winterreise ist am meisten missverstanden worden in dieser Beziehung. Weil ich da eine Form des persönlich erlebten Nihilismus beschreibe, ist mir unterstellt worden, ich beschriebe ein Programm des Nihilismus, was überhaupt nicht der Fall war: mich hat die philosophische Basis dieser Geschichte interessiert. Es ist auch doppelt interessant, dass gerade von ideologischen Seiten die meisten Einwände waren. Denn der Marxist, dem ist noch lieber der Katholik als der Nihilist und umgekehrt. Der Katholik, dem ist der Marxist auch noch lieber als der Nihilist. Was die fruihen Arbeiten betrifft, bin ich bei diesen Arbeiten von meiner eigenen Subjektivität so stark gefangen genommen, dass ich sie eben als Ausdruck meiner Situation innerhalb der Gesellschaft, in der ich gelebt habe, sehe und auch meine Beschränkung innerhalb der Gesellschaft, in der ich gelebt habe und lebe, dass ich mir sozusagen das Tarngewand des Schizophrenen angezogen habe, also meine Ver-rücktheit, meine Verschobenheit Ausdruck gefunden hat in einem ver-rückten Text.

28 Siehe Der große Horizont, S. 31-32, 115-116, 135, 154 und 172 (Suhrkamp Ausgabe). 
Auch das halte ich für eine Form von subjektiver Revolution, über die man geteilter Meinung sein kann, die mir aber in dieser Phase meiner Entwicklung als einzig mögliche Form, mich auszudrücken, zur Verfügung gestanden ist.

SR: Das war für Dich im Wienerischen Sinn ,ein Aufstand gegen die Sprache. ${ }^{629}$

GR: So ist es, auch das ist ein Aufstand gegen die Sprache, aber es war auch ein Genuss, schwer verständlich zu sein, weil ich mich auch nicht verstanden gefühlt habe. Es hat auch mit dem zu tun gehabt.

SR: Wie siehst Du denn Deine Position in der neueren österreichischen Literatur?

GR: Vielleicht kann ich nur sagen, wie ich mich verhalte, denn ich kann da keine Beurteilung vornehmen. Wenn ich arbeite, bin ich sicherlich ein Einzelgänger. Im Forum Stadtpark habe ich zwei Freunde, den Kolleritsch und den Bauer, beide machen eine andere Literatur als ich, trotzdem sind sie mir als Anwesende wichtig, die ich oft imaginär beschreibe und mir oft denke, welche Reaktion das, was ich schreibe, bei ihnen auslösen würde. Ich kann meine Position

29 Siehe O. Wiener Die Verbesserung von Mitteleuropa, S. CXLIV: „die sprache wird gemeinhin als gesellschaftliches bewusstsein, ja als gedächtnis der menschheit bezeichnet. diesen kalauer einmal wörtlich genommen: ein aufstand gegen die sprache ist ein aufstand gegen die gesellschaft." 
innerhalb des Literaturbetriebs nicht ermessen, ich bin froh, sobald ich schreiben kann, wenn ich schreibe, dass ich schreibe. Ich kann nicht sagen, dass es mich nicht interessiert, aber es beeinflusst mich nicht und ich verschwende keine Gedanken daran.

SR: Du hast aber z. B. das Stipendium von der Stadt Hamburg bekommen, was eigentlich viel über Deine Position in der Bundesrepublik sagt.

GR: Ich kann dazu nichts sagen. Ich kümmere mich nicht darum, was für eine Position ich in Österreich habe. Ich habe keine Schwierigkeiten, in Österreich schriftstellerisch tätig zu sein und ich habe auch keine Schwierigkeiten insofern, dass meine Arbeiten in Österreich nicht beachtet würden. Es ist in letzter Zeit, was die Printmedien betrifft eine gewisse Feindseligkeit oder eine größere Härte mir gegenüber vorhanden, aber ich kann das jetzt nicht untersuchen, warum das der Fall ist, sondern ich muss eben meine Sache weiter machen. Ich glaube, dass ich in Deutschland mehr Anerkennung habe als in Österreich, das stimmt aber auch wieder nicht hundertprozentig. Es sind alle meine Stïcke in Österreich aufgeführt worden, meine Bücher sind in allen Zeitungen besprochen worden, wie jetzt dann die Resultate ausfallen, ist oft zufällig. Man kann ja nicht jeden Kritiker ernst nehmen, der in einer Zeitung schreibt. Es wird ja oft zwischen Kritiker und Zeitung verwechselt. Man sagt z. B. diese Zeitung, Der Spiegel usw. hat so und so geschrieben und da sitzen auch ganz unterschiedliche Kritiker und in Österreich gibt es ja keinen Literaturkritiker, den ich ernst nehmen kann. Von Zeitungen will ich überhaupt nicht reden. 
Das Interessante ist, dass es nicht nur schlechte Tageszeitungen gibt, die im Ausland nicht reflektiert werden, die nicht zitiert werden im Ausland, weil sie nicht zitierbar sind. Wer über Österreich etwas erfahren will, kann in der Sïddeutschen und in der Neuen Zürcher Zeitung Besseres und Genaueres erfahren als in den eigenen Zeitungen.

Zweitens ist eben der Kulturjournalismus in Österreich verrottet. In Wien gibt es so einen Salonkritikerhof, wo ein paar Herren und Damen das machen, was sie unter Kultur verstehen, was alles sehr nach Freundschaft, Bekanntschaft und Intervention ausgerichtet ist. In Deutschland ist es nicht besser als in Österreich, aber sie haben bessere Zeitungen, das steht außer Frage, und offensichtlich bringt auch diese Möglichkeit für bessere Zeitungen zu arbeiten, bessere Journalisten hervor. Deshalb sind einige Zeitungen in Deutschland informativer und wichtiger für die österreichische Literatur als die gesamten österreichischen Zeitungen. Es gibt in Deutschland aber auch eine Serie von sehr schlechten Zeitungen.

SR: Aber Die Zeit z. B. oder die Frankfurter Allgemeine?

GR: Der Spiegel, Die Süddeutsche, diese Zeitungen kann man in Deutschland nennen. In der Schweiz, die Neue Züricher Zeitung, damit hat es eigentlich ein Ende.

SR: Die sind alle wichtig für die Rezeption der österreichischen Literatur.

GR: Ja, aber diese Zeitungen werden von der österreichischen Bevölkerung eben nicht gelesen. Speziell Literaturin- 
teressierte oder politisch Interessierte lesen vielleicht Die Zeit oder vielleicht die $F$. A. Z, schon die Rundschau wird weniger gelesen.

GR: Also zu der Frage Büchner und Naturwissenschaften, glaube ich, dass ich nicht wesentlich etwas hinzufügen muss, was die frühen Arbeiten betrifft. Erst wiederum im Stillen Ozean hat dieses Thema Auswirkung gehabt, das hat dann mit Büchner nichts mehr zu tun. Das ist mein Wissen, das ich eben dieser Figur angezogen habe.

SR: Helmut Eisendle versucht in seinen Romanen eine Ästhetik aus einer Mischung von Wissenschaft und Kunst $\mathrm{zu}$ machen. Wie reagierst Du auf diesen Versuch?

GR: Ich habe zumindest in der Anfangszeit seine Arbeit genauer verfolgt, doch in der letzten Zeit nicht mehr. Allerdings ist das keine Beurteilung seiner Arbeit von mir aus. Es ist einfach so, dass ich in der letzten Zeit die Bücher, aus welchen Umständen auch immer, eben nicht gelesen habe. Mir war Eisendles Arbeit phasenweise zu sehr schon an die Wissenschaft angelehnt.

Um jetzt noch einmal auf die Ideologie zurückzukommen, dann muss ich sagen, dass das mir eben schon zu sehr Wissenschaftsideologie ist, dass das Werkzeug wieder zu stark wird gegenüber der Literatur. So wie eben Ideologien eine Hilfe beim Schreiben sein können, einzelne Prozesse und Strukturen zu durchschauen und sie wieder zu transformieren, sie wieder in den literarischen Prozess hineinzuverwan- 
deln, ohne mit dem Zeigefinger aufmerksam zu machen, so erscheint es mir wichtig auch den Einfluss der Naturwissenschaften auf die Literatur, so ich es mache, in Literatur zu verwandeln, und, dass diese Ansatzpunkte nicht zu deutlich und zu klar herauskommen. Sie sollen spürbar sein aber nicht bestimmend. Das ist die kritische Distanz, die ich zu den Arbeiten von Helmut Eisendle halte.

\section{$* * *$}

SR: In Winterreise kommt eine Passage vor, ich zitiere: „Manchmal war es seine einzige Hoffnung, dass es einen Gott gäbe. ... Manchmal spürte er jedoch, dass es ihn geben musste." Und dann auf der folgenden Seite: „Er habe nur selten grundlos das Gefühl gehabt, dass es einen Gott gab. “30 Dann im Stillen Ozean klingt es ein bisschen anders: „Vielleicht war er auch nur eine winzige Zelle in einem größeren Organismus, den er nicht erkennen konnte, ja, nie begreifen würde. “ 31

GR: Ich würde meine Position so ausdrücken, dass ich im Geheimen in meinem Alltag mit meiner metaphysischen Ebene korrespondiere. Sehr gelegentlich. Sie scheint mir vernunftmäßig nicht erklärbar, ich kann keine Gründe dafür anführen, außer die Tatsache, dass es bei mir so der Fall ist. Das hat nichts mit einem bestimmten Glauben zu tun. Ich möchte z. B. das Wort ,Gott' gar nicht aussprechen. Ich verwende es nur um diese metaphysische Ebene auch punktuell anklingen zu lassen in meiner Arbeit. Für mich ist das

${ }^{30}$ Winterreise, S. 90-91.

${ }^{31}$ Der stille Ozean, S. 28. 
etwas Außersprachliches, Außerpsychologisches, aber sicherlich vom Augenblickszustand, indem ich bin, ziemlich beeinflusst.

SR: Ist das etwas, was man spüren kann?

GR: Es ist ein Gefühl, ja. Es ist eine Empfindung und oft ein Wunsch, der, wie ich es im Stillen Ozean schreibe, sobald ich ihn zu formulieren versuche, nebulos wird, einfach verschwindet.

SR: Der Ascher versucht am Anfang des Romans zu beten. Er fühlt sich ganz blöd, er kann es nicht.

GR: Ich meine mit dieser Kommunikation eine außersprachliche Kommunikation, eine nicht in Wort gefasste Situation. Es gibt eine Form des Betens in der Verzweiflung, die mir nicht fremd ist. Die findet sich z. B. mehrfach im Werk von Hemingway, wo Figuren diese Stoßgebete zum Himmel senden in Todesangst oder in Verzweiflung.

SR: Kafka hat das Schreiben selbst eine Form des Gebets genannt. $^{32}$

GR: Das Wort ,Gebet' ist mir zu nahe einem Ritual, das mit bestimmten Religionen zusammenhängt. Auch das Wort ,Meditation' ist mir bereits zu abgegriffen und zu modisch.

32 „Schreiben als Form des Gebets.“ Franz Kafka, Hochzeitsvorbereitungen auf dem Lande und andere Prosa aus dem Nachlaß, Max Brod (Hrsg.), Franz Kafka Gesammelte Werke, Taschenbuchausgabe in sieben Bänden, Frankfurt a/M.: Fischer, 1983, S. 252. 
Es ist, ich kann das nicht anders bezeichnen, eine Korrespondenz mit einer metaphysischen Ebene. Und die ist sehr oft vom Visuellen bestimmt. Zum Beispiel, ich habe eine Serie von optischen Geräten immer bei mir - Taschenmikroskope, Taschenlupen, Fernrohre, Taschenfernrohr - und es ist sehr interessant, dass gerade in solchen Momenten, wenn ich z. B. im Sommer durch eine Wiese gehe und eine Blume unter einer Lupe oder unter einem Taschenmikroskop anschaue, oder wenn ich einen Habicht in der Luft verfolge, so eine Korrespondenz möglich ist. Ich sage nicht gelingt, weil ich sie nicht beabsichtige. Ich mache das nicht deswegen, damit ich dieses Gefuihl habe, aber es entsteht bei mir dann unter solchen Voraussetzungen. Oder z. B. wenn ich in einem Teich längere Zeit die Fische beobachte. Das ist nicht jedes Mal der Fall, aber unter solchen Aspekten war es für mich der Fall und ich habe mich in der Zeit, wo Winterreise war, sehr stark mit Steiner beschäftigt, während ich diesen Roman geschrieben habe, krank war und eigentlich mich auch mit meinem Tod auseinandergesetzt habe und da war diese metaphysische Ebene sehr stark in mir. Da habe ich sehr viel über den Tod gelesen und diese Ansichten von Steiner, dem Anthroposophen, die im nüchternen Zustand betrachtet Mumpitz sind, aber in einem bestimmten geistigen Zustand, eine seltsame Anregung bilden. Ich glaube, dass man mit seinem Bewusstsein verschiedene Aggregatzustände durchläuft im Leben. Wenn ich das flüssig, fest und gasförmig für mein Seinsverhalten nehme, dann gibt es eine Schicht von sehr nüchterner Person in mir, die über lange Strecken des Lebens die wirksamste ist. Es gibt die Situation des Arbeitens, des Schaffens, des Schreibens und schließlich die des untätigen Beobachtens und Erlebens, des sehr wa- 
chen, untätigen Beobachtens und Erlebens. Und in jedem dieser verschiedenen Zustände gibt es für mich eben verschiedene Einstellungen zu diesen Dingen und ich kann diese Frage vom Religiösen her überhaupt nicht betrachten. Mir geht es auf die Nerven, wenn ich Katholiken ihren Sonntagsdienst verrichten sehe, da habe ich immer eine Abneigung davor. Oder der Papst geht mir auf die Nerven. Das hat überhaupt nichts mit so etwas zu tun. Das hat am ehesten etwas mit der buddhistischen Form von Religion zu tun, obwohl es auch mit dem Buddhismus nichts zu tun hat. Es ist nur von dort her am möglichsten zu begreifen. Ich will nicht dieses Gefühl, das ich hin und wieder empfinde in irgendetwas einordnen. Nur deswegen, weil ich es nicht sprachlich und geistig fassen kann, will ich nicht nach einer Fassung suchen, in die ich das hineinpresse. Der Zustand beim Schreiben, das heißt also beim Verwandeln, weil Schreiben im Wesentlichen für mich eine Form der Verwandlung ist, ist sicher kein realistisch nüchterner Zustand. Das hat schon Sartre erkannt, der hat zwar seine Meinungen im Laufe der Jahre oft geändert, so dass er für jedes Argument irgendwo zitierbar ist. Das hat er aber, soweit ich mich erinnere, nirgendwo widerrufen - dieses Berühren des Transzendentalen in der besten Situation des Schreibens.

SR: Es gibt bei Camus im Fremden die Szene im Gefängnis, wo der Pfarrer kommt. Meursault schickt ihn weg, dann schaut er aus dem Fenster auf die Sterne.

GR: Ja, für mich ist das begrenzt durch die Form der Ausdrucksweise und das, was Camus hier eben zeigt, die Winzigkeit der Erde, die unfassbare Ausdehnung des Raumes 
um uns, die Unfassbarkeit des Begriffs , unendlich', das gleichzeitig Trost und Verhöhnung ist, je nach dem in welchem psychologischen Zustand man ist.

SR: Wie reagierst Du auf das Wort ,Schöpfung'? Kannst Du so ein Wort benutzen oder ist das schon zu viel?

GR: Ich glaube dass dieses Wort nur in bestimmten $\mathbf{Z u}$ sammenhängen zu benutzen ist, es kommt darauf an, von welchen anderen Worten es umgeben ist. Nur dadurch, dass ich mir die Schöpfung nicht erklären kann, nur dadurch, dass es keine konkrete Erklärung für die Schöpfung gibt, heißt für mich nicht, dass dadurch zwangsläufig die Religion bewiesen ist. Das heißt für mich nur, dass eben das menschliche Gehirn, so unfassbar kompliziert und großartig es ist, zu welch großartigen Einsichten es fähig ist, eben seine Grenzen hat: Grenzen der Aufnahmefähigkeit, Grenzen der Erkenntnis. Ob das jetzt nur so ist, dass wir nur die höchstentwickelten Intelligenzen des gesamten Alls sind, oder ob das so ist, dass es noch eine höhere Intelligenz in dem All gibt, das berührt mich für mein persönliches Leben nicht. Ich würde den Tod - damit hängt das Ganze wieder zusammen - nicht als Strafe empfinden. Es ist einfach ein Verlust der Umwelt, dass man eben nicht mehr dabei ist; es ist einfach dieser Titel „Der lange Abschied“ - das ist das Furchtbare an der Geschichte. Alles andere ist ja - egal wie es nun weitergeht - nicht erschreckend für mich. Die Vorstellung des Todes als Dunkelheit hat nichts Erschreckendes für mich und die Vorstellung des Todes als eine Form der Bewusstheit auf einer anderen Ebene, hat auch nichts Erschreckendes für mich, scheint mir aber äußerst unwahrscheinlich. 
SR: Im Großen Horizont steht Haid im Friedhof in New York und hat das Gefühl, dass es möglicherweise eine andere Wirklichkeit gibt. Er beschreibt seine Erfahrung von Caspar David Friedrichs Greifswaldbild.

GR: Noch einmal, das ist so ein Zustand einer Korrespondenz mit dem Metaphysischen, wie ich ihn beispielsweise beim Betrachten von Blumen empfinde oder beim Vogelflug. Das sind einfach Momente. Das ist dann auch bald wieder verschwunden. Zurück bleibt eine Erinnerung, ein Gedanke daran, der, wenn er isoliert als solcher genommen wird, wie ich ihn jetzt beschrieben habe oder wie ich ihn erzähle, etwas ist, wozu ich mich bekenne. Aber ich kann aus diesen mikroskopischen Erinnerungen an Erfahrungen keine Struktur bilden, die mich zu einem Entschluss führt, etwas zu behaupten oder für etwas einzutreten. Ich möchte uiberhaupt nicht mehr in der Angelegenheit sagen als ich empfinde, aber ich möchte bei dem bleiben, was mir zustößt. Es wäre mir sehr unangenehm mehr dazu zu sagen. Dass ich z. B. beim Fliegen ein stummes Gebet, beim Start, sage, das habe ich auch schon gemacht. Ob das jetzt Erziehung ist, ob das in mir drinnen ist oder ob das jetzt der Ausdruck der Wahrheit ist, das kann ich gar nicht deuten, denn in dem Moment, in dem die Situation nicht mehr da ist, bin ich auch anders und es ist trotzdem richtig in dem Zustand, in dem ich mich befinde. Ich habe auch gar nicht das Gefühl, dass ich jetzt meine Haltung betrüge, wenn mir das durch den Kopf geht, ich kann mich ja nicht einfach gegen Gedanken wehren, die in mir aufstehen. Wie ich jung war, so bis 26 , 27, 28 habe ich ziemlich starke Todesängste gehabt, das ist 
in den letzten Jahren sehr stark zurückgegangen. Ich fürchte das jetzt nicht mehr.

SR: Kannst Du das erklären oder gehört das einfach zum Lauf der Zeit?

GR: Ich weiß nicht, ob das mit dem Alter zu tun hat, eigentlich wird man ja, wenn man älter wird, immer ängstlicher. Ich glaube eher, dass ich eine gewisse Bereitschaft dazu habe, zum Sterben. Ich wünsche es mir nicht, momentan, aber es gibt auch Situationen, wo ich es mir wünsche. Mein Leben verläuft nicht in so einheitlichen Linien, dass ich $\mathrm{z}$. B. immer derselben Meinung oder derselben Ansicht bin. Wenn mich jemand allgemein, oberflächlich fragen würde, „Glaubst Du an Gott?“, würde ich sagen: „Nein.“ Das ist das Erste, was ich sagen würde und dann würde ich die Gründe anführen, weshalb das nicht der Fall ist.

\section{SR: Es geht also um die Form der Frage?}

GR: Es geht um die Form der Frage, genau, welche Erfahrung jemand ansprechen will in mir. Dass ich diese Erfahrungen habe, das heißt für mich nicht, dass ich Erfahrungen mit Gott habe, aber ich würde sagen, es ist keine naturwissenschaftliche Ebene mit der ich korrespondiere und auch keine politische, sondern es ist eine Selbsterfahrung von einer großen Intensität, sprachlos, ohne sprachliche Formulierung, wie wenn man plötzlich über etwas glücklich ist und das hält auch nur einige Zeit an. Dieser Anblick verwandelt mich, bringt das Gehirn zum Flimmern irgendwo. Es ist wie ein schöpferischer Prozess, das ganze, nur dass er nicht 
schöpferisch in dem Sinne ist, dass ich mich jetzt hinsetze und schreibe, sondern dass er an und für sich mir genügt, dass die Erfahrung für mich ausreicht und ich auch kein Bedürfnis habe, mich mit ihr auseinanderzusetzen.

\section{$* * *$}

SR: Eine andere Frage, zum Vesuv, der in Winterreise vorkommt. Was bedeutet das überhaupt?

GR: Der Vulkan. Er bedeutet einen Vulkan. Ich bin beim Schreiben, nachdem diese Überlegungen über Freuds Unbehagen in der Kultur und Camus Der Fremde, getroffen waren, von einem Bild ausgegangen und dieses Bild war der Vulkan. Empedokles auf dem Vulkan - das waren einfach so Bilder, die ich hatte. Und dann habe ich in einer Buchhandlung ein Buch über Vulkane gefunden, das hat mich ungeheuer fasziniert und dann hatte ich so im Hinterkopf einen Kurzfilm von dem Wiener Filmemacher Kubelka. ${ }^{33}$ In diesem Film ist ein Vulkan zu sehen - ich weiß es nicht mehr genau, es liegt schon Jahre zurïck - vielleicht ist es der Vesuv knapp vor seinem Ausbruch, oder ist es der Ätna und da wird in diesem Haus eine Hochzeit vorbereitet, man sieht auch so erstarrte Lavamassen rund herum und seit ich das gesehen habe, hatte ich das Gefuihl, den Wunsch, etwas über einen Vulkan zu schreiben, das ist eigentlich alles. Das hat keine weitere Bedeutung. Der Winterreise werden von der Kritik symbolische Dinge unterschoben, die nicht da

${ }^{33}$ Peter Kubelka (geb. 1934), österreichischer Avantgarde-Filmemacher und Filmtheoretiker. Gründete mit Peter Konlechner 1964 das Österreichische Filmmuseum in Wien, das er bis 2001 als Direktor leitete. 
sind. Die Winterreise ist nicht symbolisch, die Winterreise ist rhythmisch, es kehren ähnliche Motive immer wieder.

SR: Ist das ein musikalisches Prinzip?

GR: Ein musikalische Prinzip, ja. Man kann auch sagen ein mathematisches Variationsprinzip. Aber die Dinge, die im Buch vorkommen, werden auch beim Namen genannt. Es gibt einige seltsame hitchcockartige Szenen, die den Tod anzeigen sollen, wie diese eine wo der Hotelangestellte in dem Augenblick hereinkommt als der Geschlechtsverkehr im Gange ist oder in der Bahnfahrt, dass die Tür geöffnet wird, aber alles andere ist vordergründig. Die Erinnerung des Engels hat keine Symbolik, das ist eben die Erinnerung eines Engels und es ist typisch, dass in der amerikanischen Kritik überhaupt keine Symbolik gesehen wurde, während in der tiefsinnigen deutschen Kritik das selbstverständlich als symbolschwer gesehen wird. Ich habe überhaupt keine symbolischen Bücher geschrieben. Es gibt auch im Großen Horizont keine symbolischen Handlungen und es gibt auch im Neuen Morgen keine symbolischen Handlungen: ich mag den Symbolismus in der Literatur überhaupt nicht. Ich kann nur von riesigen Missverständnissen sprechen. Ich meine ganz sicher kann ein bestimmter Punkt in einer Geschichte, wie z. B, der Moby Dick in Melville, auch ein Symbol sein, aber das geht nur mit einem Doppel-, Dreifach-, Vierfachboden. Zunächst ist das eine klare, einfache Geschichte, die man genauso lesen kann, wie sie dasteht. Alle anderen Möglichkeiten, die sich daraus schlüssig ergeben, sprechen dann nur für ein Buch. Man darf nicht vor lauter Möglichkeiten das Buch aus den Augen verlieren. Zunächst geht es mir 
einmal um die Geschichte, dass dort und da dann andere Deutungen möglich sind, das spricht eher für das Buch. Oft sind Deutungen möglich, die ich gar nicht beabsichtigt habe, was eine Art Psychoanalyse des Kritikers - oder bei einem Stiuck des Regisseurs - meiner Person ist. Man kann auch einen Text psychoanalysieren.

SR: Es besteht besonders bei der Winterreise die Versuchung für die Kritiker, diesen Roman psychologisch, bzw. psychoanalytisch zu interpretieren: Ein Vulkan mit Eis und Schnee drauf, die Sexualität - man spürt da eine enge Beziehung zwischen den beiden Äußerungen.

GR: Ja, weil es eben zusammenpasst. Der Titel Winterreise wurde erst am Schluss des Buches von mir geschrieben. Die Reise habe ich im Winter, nachdem der Plan festgestanden ist und ich mir sicher war, dass ich das machen will, gemacht, weil die Partnerin beruflich keine andere Möglichkeit hatte, als zu dem Zeitpunkt zu fahren, zu dem ich gefahren bin. Ich wollte aber die Reise machen, weil ich eben dieses Buch schreiben wollte und diese Stationen sehen wollte. Ich habe also diese Reise gemacht, nachdem ich vorher gewusst habe, ungefähr, was ich schreiben will.

SR: Der Zufall ist in diesem Fall ein großer Künstler.

GR: Ja, ich glaube nicht an diesen Zufall. Ich glaube, dass ich das Buch auch im Sommer bei einer großen Hitze hätten schreiben können. Dann hätte man gesagt, ja die Hitze und die Sexualität, das sind zwei Dinge, die gehören unbedingt zusammen, oder im Herbst, wo der Tod ist. Man darf das 
einfach nicht überschätzen. Weil das Buch jetzt Winterreise heißt, ist die Jahreszeit jetzt plötzlich so wichtig für die Erklärung des Buches. Ich hätte im Sommer halt einen anderen Titel gewählt.

SR: Der Titel wäre vielleicht wichtiger, wenn Du die Geschichte erfunden hättest, anstatt alles so wirklichkeitsnah zu beschreiben. Ich meine, wenn man bewusst Symbole verwendet, ist das etwas völlig Anderes.

GR: Na ja, das ist ja auch z. B. der Grund, warum der Jon$\mathrm{ke}^{34}$ bei der deutschen Kritik einen unangreifbaren Status hat, weil er nichts Angreifbares schreibt, das ist nicht vergleichbar. Aber ich kann doch nicht erwarten, wenn ich eine Erfahrung erzähle, eine Geschichte erzähle, dass Leute mit verschiedenen Weltanschauungen, mit verschiedenen Charakteren, mit verschiedenen Deformationen der Charaktere, jetzt alle sagen werden, das ist gut und die Geschichte ist richtig. Mir ist ganz klar, dass die Rezeption meiner Arbeiten auf Feindseligkeit stoßen wird, mir ist auch klar, dass ich mich oft auch auf eine herbe Kritik einstellen muss, aber ob sie berechtig ist, das wird sich erst herausstellen. Ich kann die Sicherheit der Kritiker nicht teilen. Ich bin mir ziemlich unsicher und lebe mit dieser Unsicherheit abwechslungsweise trotzig, verletzt, oft der Zerstörung nahe, sehr oft mit Selbstmordgedanken, dann wiederum sehr sicher meiner Sache, weil der Kritiker eben sein Urteil über das Buch abgibt und dann hat sich's für ihn. Ich lebe mit dem, das ist mein Leben, das ist meine Dauerbeschäftigung.

${ }^{34}$ Gert Jonke (1946-2009), österreichischer Schriftsteller und Dramatiker, Mitglied der Grazer Autorenversammlung. 
SR: Wie wichtig ist es für Dich, dass Deine Romane und Theaterstuicke verstanden werden?

GR: Jeder Mensch will verstanden werden, auch der Wahnsinnige will verstanden werden, auch wenn er sich eine eigene Sprache zurechtlegt. Wenn ich nicht mehr verstanden werden will, dann schweige ich. Wenn ich nicht schweige, sondern spreche oder male oder etwas herzeige, dann will ich verstanden werden. Und es ist sicher etwas Angenehmes von möglichst Vielen verstanden zu werden.

SR: Du könntest z. B. nicht die Haltung Stendhals akzeptieren, dass man mindestens zur Lebenszeit nicht erwarten kann, vom Publikum verstanden zu werden.

GR: Bei solchen Äußerungen ist meist auch etwas Koketterie dabei.

SR: Das hat Hans Wollschläger auch vor kurzem gesagt. Er hat eine fatalistische Position adoptiert. Es stört ihn nicht, dass niemand seine Werke versteht.

GR: Das wäre eben die Betrachtung der Arbeit als Selbstzweck. Ich bewundere es, wenn jemand diese Konsequenz hat, aber es ist noch kein Zeichen von Qualität so zu denken. Es haben z. B. Kleist und andere sehr große Sehnsucht gehabt, verstanden, gespielt und anerkannt zu werden. Das man es tut $-d$. h. seine Sachen weiter schreibt, auch wenn man nicht anerkannt wird - das ist eine andere Sache oder wenn man keine Mitteilung hat. Das heißt diese Besessenheit zur Arbeit zu haben und sie nicht anders zu können als 
so, wie man sie macht. Wenn ich keine Erwartung habe, verstanden zu werden, heißt das ja nicht, dass es mir egal ist, ob ich verstanden werde oder nicht. Ich kann in diesem Fall nur sagen, ich muss das so machen, wie ich es tue und rechne nicht mit einem Verständnis, so wie jemand, der etwas Gutes tut nicht mit dem Dank rechnet, sondern es um des guten Willens tut. Aber dass prinzipiell der Wunsch nach Kommunikation bei der künstlerischen Arbeit vorhanden ist, an das glaube ich. Dass der Ausdruck eine Mitteilung ist, dass es eine Form von Sprechen ist, alles, was Kunst ist, ist eine Form von Sprechen. Und ich spreche ja nicht nur einen Monolog mit mir selber, sondern ich spreche ja auch zu Menschen. Ich habe das schon einmal erzählt, die Situation meiner Kindheit, dass ich Bücher nur für mich gezeichnet und geschrieben habe. Ich kann das nur mit dieser Situation vergleichen. Bei mir war das so, dass ich diese Bücher überhaupt niemand anderen lesen lassen wollte und auch gar nicht herzeigen wollte. Da ging es mir um die Bücher an und für sich, für mich selber.

SR: Was sind für Dich die wichtigsten Zeichen dieses Verstandenwerdens? Wie weißt Du, dass Deine Bücher verstanden werden?

GR: Ich kann das nicht in der Mehrzahl sagen, was ich jetzt sage, wie viele Leute mich verstehen, oder ob es Viele sind, die mich verstehen. Aber in so einem Gespräch, wie wir es jetzt führen, habe ich den Eindruck verstanden zu werden. Und das ist mir angenehm. Oder wenn ich mit jemanden $\ddot{\mathrm{u}}-$ ber ein bestimmtes Buch, uiber eine bestimmte Figur spreche und ich merke, dass er Interesse hat und die verstanden hat 
oder wenn jemand etwas darüber schreibt oder wenn im Theater ein Stück aufgeführt wird und ich kann beim $\mathrm{Zu}$ schauen bei der Theaterarbeit bemerken wie manche Schauspieler das begreifen, was ich gedacht habe, ohne dass ich in irgendeiner Form einzugreifen brauche. Oder wie Formen des Wiedererkennens da sind bei fremden Menschen, die sich plötzlich in meinem Buch in irgendeiner Situation wieder erkennen. Ich werde auch oft verstanden, indem man sich über mich ärgert, da merke ich manchmal auch ein bestimmtes Verständnis heraus, so wie man sich selber bei irgendetwas ertappt.

SR: Wie empfindest Du das Verhalten der Kritiker? Die können einem viel Schaden anrichten.

GR: Ich bin nicht darüber hinweg, dass ich mir jetzt sage, dass mir die Kritik egal ist. Das würde nicht den Tatsachen entsprechen. Aber ich starre nicht mehr, wie zu Anfang meiner Schreibarbeit, wie ein hypnotisiertes Karnickel auf die Schlange, was da drinnen steht, sondern es hängt davon ab. Wenn ich z. B, eine schlechte Kritik zerreiße und wegwerfe, dann existiert sie tatsächlich nicht mehr für mich. Manchem Kritiker gönne ich es, dass er sich über mich ärgert, das erheitert mich irgendwo und manches ist so dumm, dass es gar keine Wirkung hat. Da gibt es natülich schon Dinge, die eine Wirkung haben. Ich hatte z. B. einmal eine negative Kritik, da bin ich drei Tage im Bett gelegen, habe Scham gespürt hinten auf die Straße hinauszugehen, Freunde zu treffen oder Menschen vom literarischen Betrieb zu begegnen, von denen man ganz genau weiß, dass sie das gelesen haben und einem das gönnen. Trotzdem, jetzt nach 
acht oder neun Jahren Schreibens akzeptiere ich es vollständig, weil sie eben auch Mitausdruck meines Scheiterns sind, von dem ich überzeugt bin und von dem ich weiß, dass es zur Arbeit dazu gehört. Ich kann nicht etwas Literarisches machen, das - zumindest wie ich es glaube - etwas Neues ist oder etwas schwer Vergleichbares ist zum Gesamtausdruck - und nur erwarten, dass es nun von allen Seiten gutgeheißen wird. Das wäre ja auch eine falsche Einstellung zu meiner Arbeit. Ich schreibe ja nicht, um von den Kritikern verstanden zu werden, sondern um das in die Welt hineinzusetzen - um es pathetisch auszudrücken - was mir wichtig erscheint.

SR: Sind die Artikel, die Du u. a. für Die Zeit bzw. Zeitmagazin geschrieben hast, möglicherweise eine Strategie die Kritiker zu vermeiden und dadurch direkt mit der Öffentlichkeit zu kommunizieren?

GR: Habe ich richtig verstanden, dass Du fragst, ob die Zeitungsartikel, die ich geschrieben habe, eine direkte Form der Kommunikation mit dem Leser sind?

SR: Ja.

GR: Die Artikel, die ich für die Zeitungen geschrieben habe, sind einerseits für mich formale Zwangsjacken in einer einfachen Form in einer Tageszeitung ein bestimmtes Problem behandelt zu haben und zweitens sind sie genau das, was Du als Frage mir gestellt hast, sind sie exakt Beispiele, die ich gebe, wenn ich über die kritische Arbeit der Kritiker oder Journalisten schreibe. Ich wollte auch in dem Metier 
arbeiten, um etwas hinzustellen, was ebenfalls angreifbar ist, wo man wieder selbst sagen kann, aber Du machst das so um das einer gewissen Tendenz des Journalismus entgegenzustellen. Natürlich sind Vorteile für mich dabei, das ich mir z. B. mehr Zeit genommen habe oder so etwas, aber wir müssen jetzt davon ausgehen, dass man nicht alles vom kleinen Alltagsjournalismus her sehen darf, was in den Zeitungen geschieht. Also z. B. in der Zeit oder im Literaturteil der F.A.Z. oder der Süddeutschen nimmt man an, dass doch etwas mehr Sorgfalt und Zeit vorhanden sein müsste als in einer kleinen Zeitung oder einer österreichischen Provinzzeitung, wo die Arbeit des Journalisten mehrfach gefächert ist. 